\title{
Effect of crosslinker on the swelling and adsorption properties of cationic superabsorbent
}

\author{
TARUN SHARMA and GIRIDHAR MADRAS* \\ Department of Chemical Engineering, Indian Institute of Science, Bangalore 560 012, India
}

MS received 8 July 2015; accepted 23 January 2016

\begin{abstract}
In the present study, superabsorbents (SAPs) of cationic monomer [2-(methacryloyloxy) ethyl] trimethylammonium chloride have been prepared by free radical solution polymerization with different crosslinkers. They were subjected to repeated cycles of swelling and de-swelling in deionized water and NaCl solution. The conductivity of the swelling medium was measured and related to the swelling/de-swelling characteristics of the SAPs. The swelling capacity was also determined in saline solution. The swelling and de-swelling processes were described by first-order kinetics. The SAPs exhibited varied swelling capacity for crosslinkers of the same functionality as well as different functionality. The SAPs were used to adsorb the dye Orange G at different initial concentrations of the dye. The equilibrium adsorption data followed the Langmuir adsorption isotherms. The SAPs were also used to adsorb three other dyes, namely, Congo red, Amido black and Alizarin cyanine green. They exhibited different adsorption capacities for different dyes. The adsorption phenomenon was found to follow first-order kinetics.
\end{abstract}

Keywords. Crosslinker; hydrogels; swelling; adsorption; kinetics.

\section{Introduction}

Hydrogels can be defined as crosslinked polymeric materials that can absorb water manifolds more than their own weight. If they absorb water more than hundred times their own weight, then they are known as superabsorbents (SAPs) [1]. They comprise of hydrophilic groups, which results in the absorption of water while the crosslinks prevent the complete dissolution of the polymer in water, and are used in a wide variety of applications [2]. The foremost use comes directly from their defining property (i.e., absorption of a large amount of water) in the form of personal hygiene products [3], such as diapers, female sanitary napkins, adult incontinence products and as a material for improving the water retention capacity of soil [4]. The SAPs made of ionic monomeric units can be used to adsorb oppositely charged species like heavy metal ions and dyes from waste water [5,6].

The swelling and de-swelling can be altered by varying the external environment in the form of $\mathrm{pH}$, temperature, salinity, etc. The swelling behaviour of the SAPs is governed by several factors that include the type and concentration of the monomer, concentration of initiator, reactivity, functionality and concentration of the crosslinker. Several studies have reported the effect of the nature and amount of crosslinker on the swelling capacity of the hydrogels [7-10]. The effect of tetrafunctional crosslinkers such as $N, N$-methylene bisacrylamide (MBA) and ethylene glycol dimethacrylate (EGDMA) on acrylic-based SAPs has been examined and it was found that the polymers showed similar swelling

*Author for correspondence (giridhar@chemeng.iisc.ernet.in,
giridharmadras@gmail.com) capacity [7]. However, the rate of swelling was considerably higher for polymers crosslinked with MBA. In the same study [7], it was also found that the self-crosslinked polymers showed lower swelling capacity and higher swelling rate than the other polymers. The water absorption increased with an increase in the crosslinker concentration upto a certain value and then decreased. The effect of tetrafunctional MBA and octafunctional crosslinker bis(diallyl acetal) on the swelling and network parameters of hydrogels made of $\mathrm{N}$-isopropyl acrylamide and acrylic acid has been studied [8]. The polymers crosslinked with the octafunctional crosslinker showed higher swelling capacity and lower crosslinking efficiency than those crosslinked with the tetrafunctional crosslinker. Poly(acrylic acid-co- $N$-isopropylacrylamide) has been synthesized with two crosslinkers, tetrafunctional MBA and hexafunctional melamine triacrylamide (MTA) [9] with the polymers crosslinked with MTA showing higher swelling ratios. Hydrogels comprising of $N$-vinyl-2-pyrrolidone and butyl acrylate have been prepared by $\gamma$-irradiation and crosslinked by tetrafunctional MBA and hexafunctional trimethylpropane trimethacrylate (TMPTA) [10]. The polymers crosslinked with hexafunctional crosslinker showed higher swelling capacity than those crosslinked with the tetrafunctional crosslinker. These studies indicate that the functionality and nature of the crosslinker play an important role. There is no direct relation between the functionality of the crosslinker and swelling capacity of the hydrogels.

There have been many studies investigating the removal of toxic species, metal ions [11,12], dyes [13-16] and organic matter [17] from waste water using hydrogels. Poly (acrylamide-co-maleic acid) hydrogels using two different crosslinkers, 1, 4 butanediol dimethacrylate and trimethyl 
propane triacrylate, have been used to adsorb a cationic dye [15], but the crosslinker had no effect on the dye adsorption of the material.

Although there is significant literature on anionic SAPs, there are relatively few studies on cationic SAPs. In this study, the polymer with [2-(methacryloyloxy) ethyl] trimethyl ammonium chloride (METAC) as the cationic monomer was synthesized by various crosslinkers including MBA [18]. Though there are sporadic studies on the use of different crosslinkers for synthesis, there is no systematic study on the effect of different functionalities of the crosslinker on the properties of the synthesized hydrogel. The effect of crosslinkers of different functionality on the swelling, de-swelling and dye adsorption characteristics of the polymer METAC was investigated in this study.

\section{Experimental}

\subsection{Materials}

All the chemicals were used as it is without any processing. The monomer[2-(methacryloyloxy) ethyl] trimethyl ammonium chloride (METAC, $\mathrm{C}_{9} \mathrm{H}_{18} \mathrm{CINO}_{2}, \mathrm{CAS}$ : 5039-78-1) crosslinkers; ethylene glycol dimethacrylate (EGDMA, $\mathrm{C}_{10} \mathrm{H}_{14} \mathrm{O}_{4}$, CAS: 97-90-5, tetrafunctional), trimethylol propane triacrylate (TMPTA, $\mathrm{C}_{15} \mathrm{H}_{20} \mathrm{O}_{6}$, CAS: 15625-89-5, hexafunctional), pentaerythritol triacrlylate (PETA, $\mathrm{C}_{14} \mathrm{H}_{18} \mathrm{O}_{7}$, CAS: 3524-68-3, hexafunctional) and pentaerythritol tetraacrylate (PETTA, $\mathrm{C}_{17} \mathrm{H}_{20} \mathrm{O}_{8}$, CAS: 4986-89-4); and accelerator $N, N, N^{\prime}, N^{\prime}$-tetramethyl ethylene diamine (TMED, $\mathrm{C}_{6} \mathrm{H}_{16} \mathrm{~N}_{2}$, CAS: 110-18-9) were obtained from Sigma Aldrich, St Louis, MO, USA. The crosslinker, $N, N$-methylene bisacrylamide (MBA, $\mathrm{C}_{7} \mathrm{H}_{10} \mathrm{~N}_{2} \mathrm{O}_{2}$, CAS:79-06-1), dyes Orange $\mathrm{G}$ (OG, $\mathrm{C}_{16} \mathrm{H}_{10} \mathrm{~N}_{2} \mathrm{Na}_{2} \mathrm{O}_{7} \mathrm{~S}_{2}$, C.I. No.: 16230, monoazo), Congo red (CR, $\mathrm{C}_{32} \mathrm{H}_{22} \mathrm{~N}_{6} \mathrm{Na}_{2} \mathrm{O}_{6} \mathrm{~S}_{2}$, C.I. No.: 22120, diazo) and Amido black (AB, $\mathrm{C}_{22} \mathrm{H}_{14} \mathrm{~N}_{6} \mathrm{Na}_{2} \mathrm{O}_{9} \mathrm{~S}_{2}$, C.I. No.: 20470, diazo) were purchased from S.D. Fine

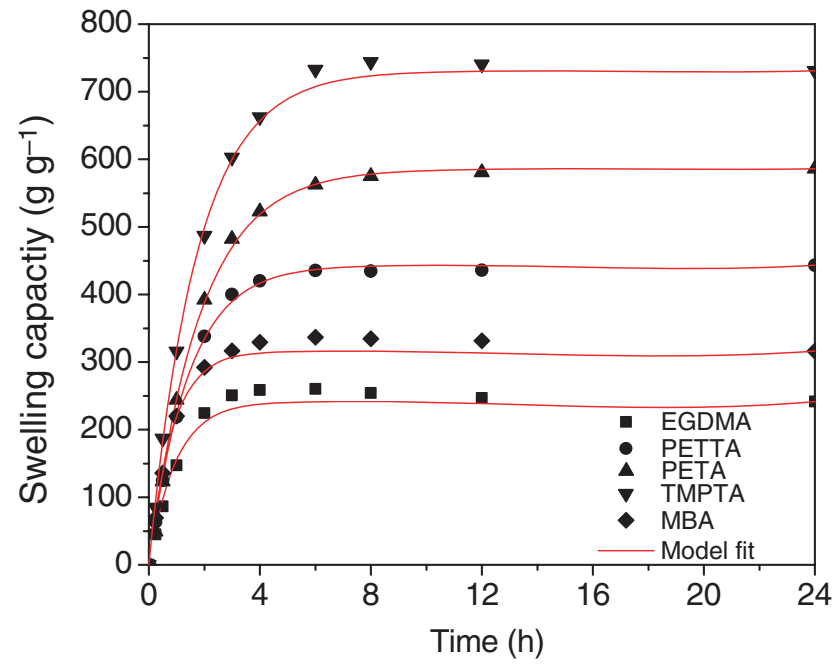

Figure 1. Variation in swelling capacity in DI water with time.
Chemicals, Mumbai, India. The dye Alizarine cyanine green (ACG, $\mathrm{C}_{28} \mathrm{H}_{20} \mathrm{~N}_{2}$, C.I. No.: 61570, anthroquinonic) was purchased from Rolex Chemical Industries, Mumbai, India. The
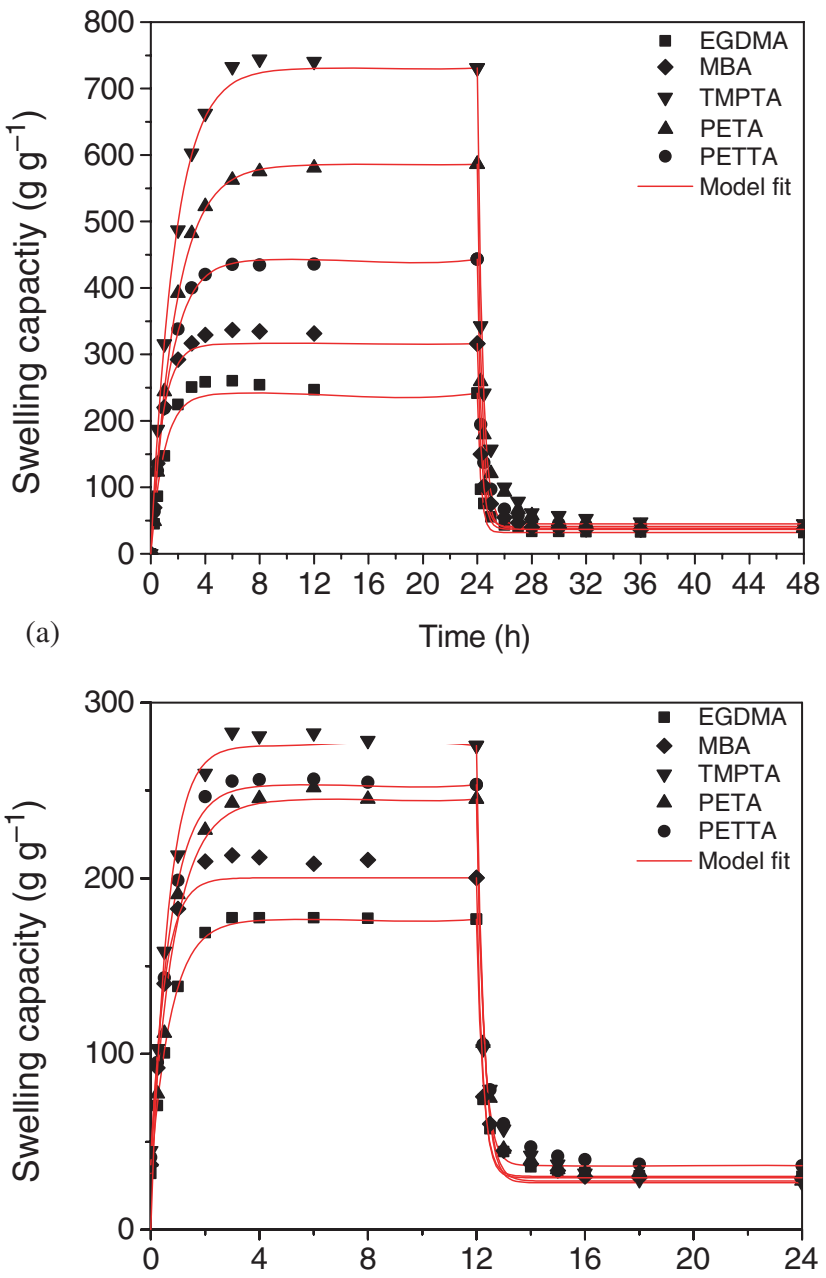

(b)

Time (h)

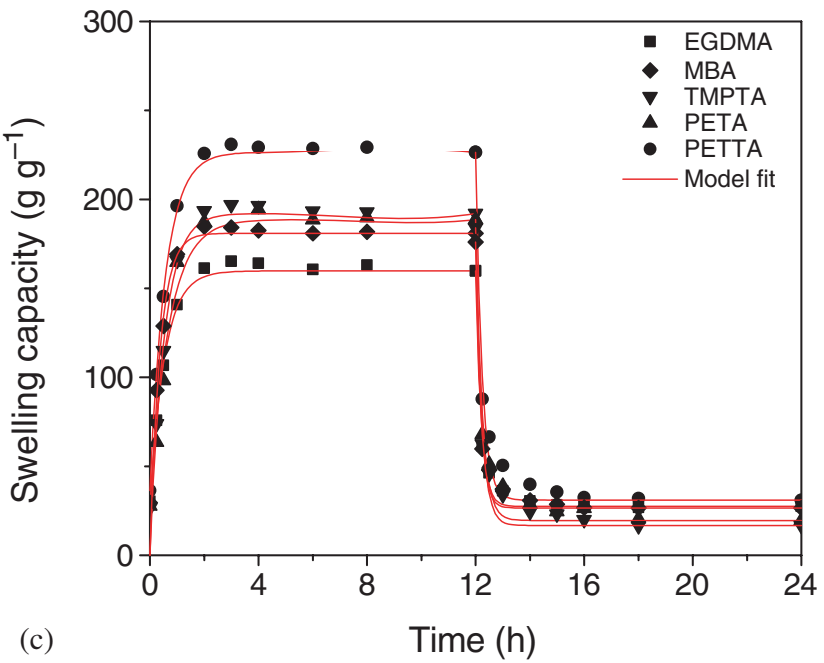

Figure 2. Variation in the swelling capacity during swelling and de-swelling cycles: (a) 1, (b) 2 and (c) 3 . 
Table 1. Parameters for swelling cycles 1,2 and 3 for different polymers.

\begin{tabular}{lcccccccc}
\hline & \multicolumn{2}{c}{ Swelling cycle 1 } & & \multicolumn{2}{c}{ Swelling cycle 2 } & & \multicolumn{2}{c}{ Swelling cycle 3 } \\
\cline { 2 - 3 } Polymer & $S_{\text {eq }}\left(\mathrm{g} \mathrm{g}^{-1}\right)$ & $k_{\mathrm{S}}\left(\mathrm{h}^{-1}\right)$ & & $S_{\mathrm{eq}}\left(\mathrm{g} \mathrm{g}^{-1}\right)$ & $k_{\mathrm{s}}\left(\mathrm{h}^{-1}\right)$ & & $S_{\text {eq }}\left(\mathrm{g} \mathrm{g}^{-1}\right)$ & $k_{\mathrm{s}}\left(\mathrm{h}^{-1}\right)$ \\
\hline MBA & $316 \pm 21$ & $1.17 \pm 0.08$ & & $200 \pm 17$ & $1.98 \pm 0.20$ & & $181 \pm 16$ & $2.43 \pm 0.29$ \\
EGDMA & $240 \pm 23$ & $1.03 \pm 0.10$ & & $176 \pm 19$ & $1.32 \pm 0.03$ & & $160 \pm 15$ & $1.84 \pm 0.08$ \\
TMPTA & $730 \pm 26$ & $0.57 \pm 0.01$ & & $275 \pm 18$ & $1.66 \pm 0.18$ & & $192 \pm 15$ & $1.78 \pm 0.17$ \\
PETA & $586 \pm 11$ & $0.54 \pm 0.01$ & & $245 \pm 17$ & $1.11 \pm 0.08$ & & $189 \pm 18$ & $1.41 \pm 0.13$ \\
PETTA & $443 \pm 21$ & $0.70 \pm 0.01$ & & $250 \pm 17$ & $1.34 \pm 0.05$ & & $230 \pm 15$ & $1.76 \pm 0.05$ \\
\hline
\end{tabular}

Table 2. Parameters for de-swelling cycles 1, 2 and 3 for different polymers.

\begin{tabular}{|c|c|c|c|c|c|c|}
\hline \multirow[b]{2}{*}{ Polymer } & \multicolumn{2}{|c|}{ De-swelling cycle 1} & \multicolumn{2}{|c|}{ De-swelling cycle 2} & \multicolumn{2}{|c|}{ De-swelling cycle 3} \\
\hline & $S_{\mathrm{L}}\left(\mathrm{g} \mathrm{g}^{-1}\right)$ & $k_{\mathrm{d}}\left(\mathrm{h}^{-1}\right)$ & $S_{\mathrm{L}}\left(\mathrm{g} \mathrm{g}^{-1}\right)$ & $k_{\mathrm{d}}\left(\mathrm{h}^{-1}\right)$ & $S_{\mathrm{L}}\left(\mathrm{g} \mathrm{g}^{-1}\right)$ & $k_{\mathrm{d}}\left(\mathrm{h}^{-1}\right)$ \\
\hline MBA & $36.9 \pm 1.1$ & $3.10 \pm 0.25$ & $29.5 \pm 2.2$ & $4.45 \pm 0.47$ & $26.5 \pm 2.4$ & $5.29 \pm 0.52$ \\
\hline EGDMA & $31.9 \pm 3.6$ & $3.87 \pm 0.39$ & $30.3 \pm 4.8$ & $4.14 \pm 0.39$ & $27.4 \pm 5.3$ & $4.88 \pm 0.35$ \\
\hline TMPTA & $44.9 \pm 2.1$ & $2.66 \pm 0.25$ & $26.6 \pm 3.1$ & $3.73 \pm 0.48$ & $16.7 \pm 2.2$ & $4.44 \pm 0.49$ \\
\hline PETA & $38.5 \pm 4.4$ & $2.95 \pm 0.31$ & $27.6 \pm 2.3$ & $3.52 \pm 0.27$ & $19.5 \pm 2.1$ & $4.21 \pm 0.47$ \\
\hline PETTA & $41.1 \pm 0.4$ & $3.18 \pm 0.29$ & $36.4 \pm 0.4$ & $3.88 \pm 0.39$ & $31.0 \pm 2.4$ & $4.23 \pm 0.40$ \\
\hline
\end{tabular}

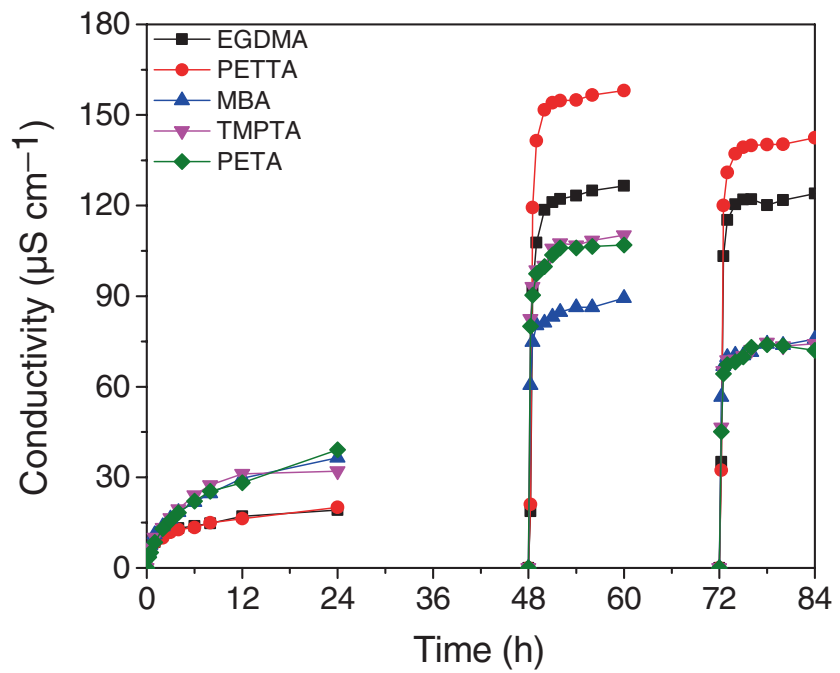

Figure 3. Variation in the conductivity of the swelling medium during swelling cycles 1, 2 and 3 of MBA and TMPTA.

initiator ammonium peroxodisulphate (APS, $\left(\mathrm{NH}_{4}\right)_{2} \mathrm{~S}_{2} \mathrm{O}_{8}$, CAS: 7727-54-0) was bought from Merck Specialities, Mumbai, India. Deionized water (DI) was used for all the experiments.

\subsection{Preparation of the SAPs}

The SAPs were prepared by free radical solution polymerization of monomer METAC with crosslinkers of different functionalities. The polymerization was carried out in an aqueous medium with a redox initiator system, APS/TMED,

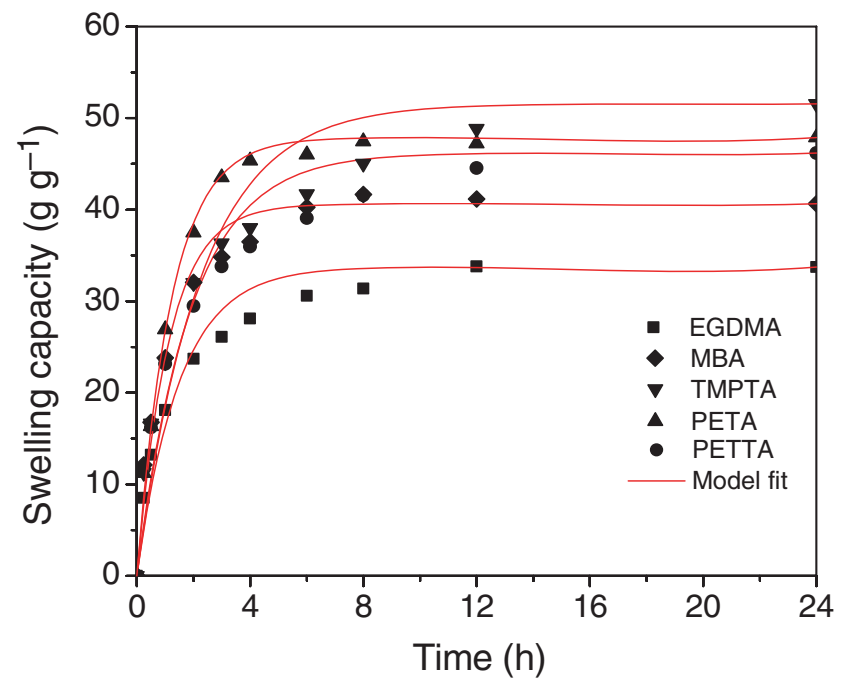

Figure 4. Variation of swelling capacity in $0.1 \mathrm{M} \mathrm{NaCl}$ solution.

where APS is the initiator while TMED is the accelerator. APS, TMED and MBA are soluble in water, while EGDMA, TMPTA, PETA and PETTA are soluble in ethanol. Stock solutions of the crosslinkers and accelerator were prepared. Quantities of 5 vol\% solutions of the following crosslinkers were prepared in ethanol: EGDMA, TMPTA, PETA and PETTA. Two wt/vol\% aqueous solution of MBA was prepared. One vol\% solution of TMED was made in DI water.

A quantity of $5.5 \mathrm{~g}$ of monomer ( $80 \mathrm{wt} \%$ solution in water) was taken in a $25 \mathrm{ml}$ beaker. The required amount of crosslinker solution was added under continuous stirring. A quantity of $0.025 \mathrm{~g}(0.5 \mathrm{~mol} \%$ of the monomer $)$ of APS 
was added and then appropriate amount of water was added so that the concentration of monomer in the reaction mixture was $45.6 \mathrm{wt} \%$. The initiator was allowed to be completely dissolved in water. Finally, $2.49 \mathrm{ml}$ of TMED solution $(0.775$ mol\% of the monomer) was added to the reaction mixture and later allowed to polymerize for $24 \mathrm{~h}$ at room temperature. The obtained polymer was washed two times in DI water for 5 min to remove unreacted components. The washed product was then dried in a hot air oven at $70^{\circ} \mathrm{C}$.

\subsection{Swelling and de-swelling}

The five kinds of polymers based on the different crosslinkers were subjected to three cycles of swelling and de-swelling. The polymers will be, hereby, referred to by the name of their crosslinker as MBA, EGDMA, TMPTA, PETA and PETTA. The swelling was carried out in DI water, while de-swelling was carried out in $0.1 \mathrm{M} \mathrm{NaCl}$ solution.

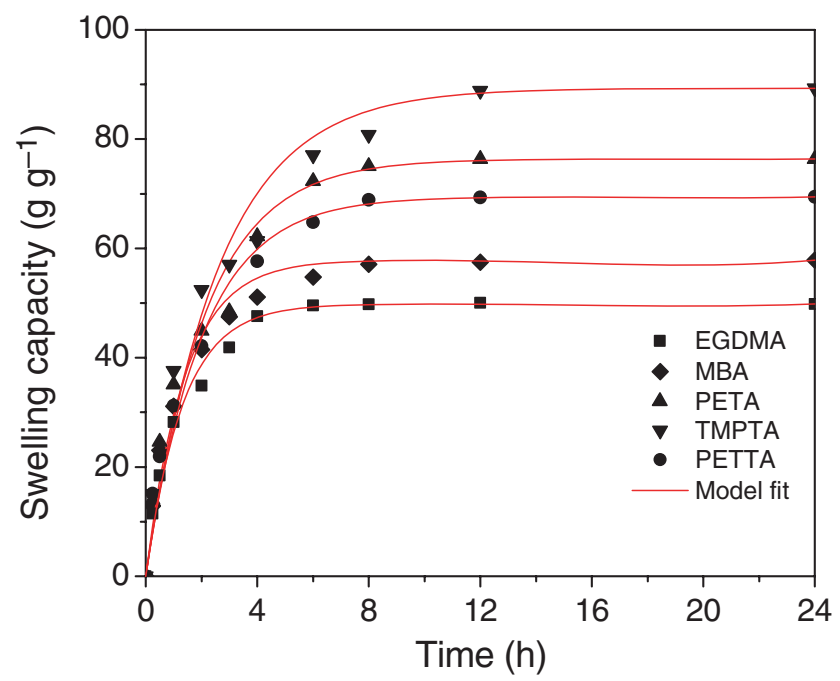

Figure 5. Variation of swelling capacity in $0.05 \mathrm{M} \mathrm{NaCl}$ solution. 2.3a First swelling and de-swelling cycle: A quantity of $0.1( \pm 0.0020) \mathrm{g}$ of polymer was taken in a pre-weighed basket made of Nylon mesh. A volume of $400 \mathrm{ml}$ of DI water was taken in a $500 \mathrm{ml}$ beaker. The basket containing the polymer was placed in the beaker. At regular intervals of time, the basket was taken out and wiped with tissue in an identical manner to remove excess water and later weighed. This was done for $24 \mathrm{~h}$. At the end of $24 \mathrm{~h}$, the basket was immersed in a $500 \mathrm{ml}$ beaker containing about $400 \mathrm{ml}$ of $\mathrm{NaCl}$ solution for de-swelling. It was weighed at regular intervals of time in the same way as described above. The de-swelling was also carried out for $24 \mathrm{~h}$.

2.3b Second swelling and de-swelling cycle: At the end of the $24 \mathrm{~h}$ of first de-swelling cycle, the polymer was subjected to the second cycle of swelling with an intermediate measurement of weights as in the first cycle. The duration of this cycle was $12 \mathrm{~h}$, after which the second de-swelling cycle was carried out in the same manner as the previous cycle for $12 \mathrm{~h}$.

2.3c Third swelling and de-swelling cycle: At the end of the second de-swelling cycle, the third swelling and deswelling cycle was carried out in the same way as the second cycle, as described above.

Apart from the above-mentioned swelling and de-swelling cycles, certain studies were done just for the measurement of swelling capacity. The procedure was same as described for the first swelling cycle.

\subsection{Equilibrium swelling study}

A quantity of $0.1( \pm 0.0020) \mathrm{g}$ of polymer was taken in the Nylon basket and dipped in about $400 \mathrm{ml}$ of saline solution. The basket was dried and weighed at regular intervals of time.

Table 3. Comparison between the swelling capacity and the limiting swelling capacity in $0.1 \mathrm{M} \mathrm{NaCl}$ solution.

\begin{tabular}{lccccc}
\hline Polymer & EGDMA & MBA & TMPTA & PETA & PETTA \\
\hline$S_{\mathrm{eq}}\left(\mathrm{g} \mathrm{g}^{-1}\right)$ & $34 \pm 2$ & $40 \pm 1$ & $52 \pm 2$ & $48 \pm 3$ & $46.2 \pm 0.7$ \\
$S_{\mathrm{L}}\left(\mathrm{g} \mathrm{g}^{-1}\right)$ & $32 \pm 4$ & $37 \pm 1$ & $45 \pm 2$ & $38.5 \pm 4.4$ & $41.1 \pm 0.4$ \\
$k_{\mathrm{s}}\left(\mathrm{h}^{-1}\right)$ & $0.7 \pm 0.07$ & $0.88 \pm 0.07$ & $0.44 \pm 0.05$ & $0.82 \pm 0.03$ & $0.52 \pm 0.07$ \\
\hline
\end{tabular}

Table 4. Swelling capacity of the polymers in $0.05 \mathrm{M} \mathrm{NaCl}$ solution.

\begin{tabular}{lccccc}
\hline Polymer & EGDMA & MBA & TMPTA & PETA & PETTA \\
\hline$S_{\mathrm{eq}}\left(\mathrm{g} \mathrm{g}^{-1}\right)$ & $50 \pm 2$ & $58 \pm 3$ & $89 \pm 2$ & $76 \pm 2$ & $69 \pm 2$ \\
$k_{\mathrm{s}}\left(\mathrm{h}^{-1}\right)$ & $0.751 \pm 0.049$ & $0.72 \pm 0.06$ & $0.36 \pm 0.04$ & $0.41 \pm 0.04$ & $0.45 \pm 0.04$ \\
\hline
\end{tabular}




\subsection{Sol content}

Sol content refers to the soluble content of the polymer. To determine the sol content, $0.1( \pm 0.0020) \mathrm{g}$ of polymer $(W)$ was taken and immersed in a beaker containing about $400 \mathrm{ml}$ of DI water and kept for 2 days. Then it was filtered and kept for drying in a hot air oven at about $70^{\circ} \mathrm{C}$ until it completely dried. Then the sample was weighed $\left(W_{\mathrm{s}}\right)$. Sol fraction, SF, is given by

$$
\mathrm{SF}=\frac{W-W_{\mathrm{s}}}{W}
$$

\subsection{Conductivity}

During the swelling and de-swelling cycles as described in section 2.3 , the conductivity of the medium was also measured using the Eutec $11+$ conductivity meter. The swelling medium was manually stirred and the conductivity meter was dipped into it and the reading shown was noted. The measurement was done prior to taking weight of the basket each time.

\subsection{Dye adsorption}

The adsorption of Orange $\mathrm{G}(\mathrm{OG})$, Congo red (CR), Alizarin cyanine green (ACG) and Amido black (AB) dyes was done in batch experiments. Dye solution of required concentration

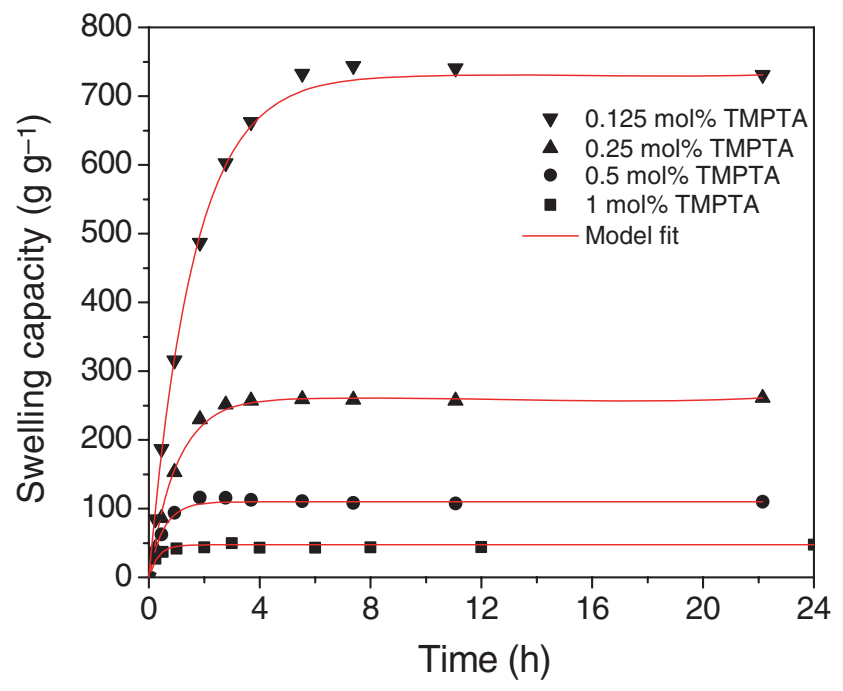

Figure 6. Variation in swelling capacity for TMPTA polymer with varying crosslinker concentrations. was prepared and $400 \mathrm{ml}$ of the solution was taken in 500 $\mathrm{ml}$ beakers. A quantity of $0.1( \pm 0.0020) \mathrm{g}$ of polymer was weighed and transferred to the beakers containing the dye solution, either in unswollen or swollen form. The concentration of the solution was determined by spectrophotometry. The absorbance by the dye solution at the wavelengths of 480 $\mathrm{nm}(\mathrm{OG}), 495 \mathrm{~nm}(\mathrm{CR}), 618 \mathrm{~nm}(\mathrm{AB})$ and $640 \mathrm{~nm}$ (ACG) was measured by Shimadzu UV-1700 PharmaSpec UV-Vis spectrophotometer and the spectra were recorded using UV Probe 2.31 software. The absorbance was converted to concentration using the calibration based on Beer Lambert's law.

The dye adsorption was quantified by using the following equations:

$$
\begin{gathered}
q=\frac{\left(C_{0}-C\right) V}{W}, \\
q_{\mathrm{e}}=\frac{\left(C_{0}-C_{\mathrm{e}}\right) V}{W} .
\end{gathered}
$$

where $q$ is the dye adsorption capacity of the polymer, i.e., weight of the dye adsorbed per unit weight of the polymer, $q_{\mathrm{e}}$ the equilibrium dye adsorption capacity, i.e., the weight of the dye adsorbed per unit weight of the polymer at equilibrium, $C_{0}$ the initial concentration of the solution, $C$ the concentration of the solution at some time, $C_{\mathrm{e}}$ the concentration of the dye solution at equilibrium, $V$ the volume of the dye solution and $W$ the weight of the polymer.

\section{Results and discussion}

\subsection{Equilibrium swelling capacity}

The polymer poly(METAC) consists of ionic monomeric units, in which the ammonium ions are the fixed entities while the chloride ions are mobile. The presence of fixed ions in the repeating unit creates a repulsive force that causes the polymer network to stretch. The polymer contains hydrophilic groups. When the polymer is immersed in DI water or aqueous solutions, the water molecules show affinity towards the polymeric units. The presence of mobile chloride ions in the polymeric network creates osmotic pressure difference which drives the water into it [19]. The crosslinks present between the macromolecular chains prevent the dissolution of polymer in water. The osmotic force makes the polymer expand by the inward movement of water while the crosslinks are retractive in nature. The polymer reaches its equilibrium swelling capacity when these forces are balanced [19]. The crosslinks hold the polymer together and keep it as a separate entity from the medium in which it is immersed.

Table 5. Parameters for swelling of TMPTA polymer with varying crosslinker concentrations.

\begin{tabular}{lcccc}
\hline Conc. of crosslinker & $0.125 \mathrm{~mol} \%$ & $0.25 \mathrm{~mol} \%$ & $0.5 \mathrm{~mol} \%$ & $1 \mathrm{~mol} \%$ \\
\hline$S_{\mathrm{eq}}\left(\mathrm{g} \mathrm{g}^{-1}\right)$ & $\begin{array}{r}730.9 \pm 26.5 \\
0.57 \pm 0.01\end{array}$ & $\begin{array}{c}261.0 \pm 11 \\
k_{\mathrm{S}}\left(\mathrm{h}^{-1}\right)\end{array}$ & $\begin{array}{c}110.0 \pm 5.1 \\
1.75 \pm 0.04\end{array}$ & $\begin{array}{c}47.5 \pm 0.5 \\
3.07 \pm 0.39\end{array}$ \\
\hline
\end{tabular}




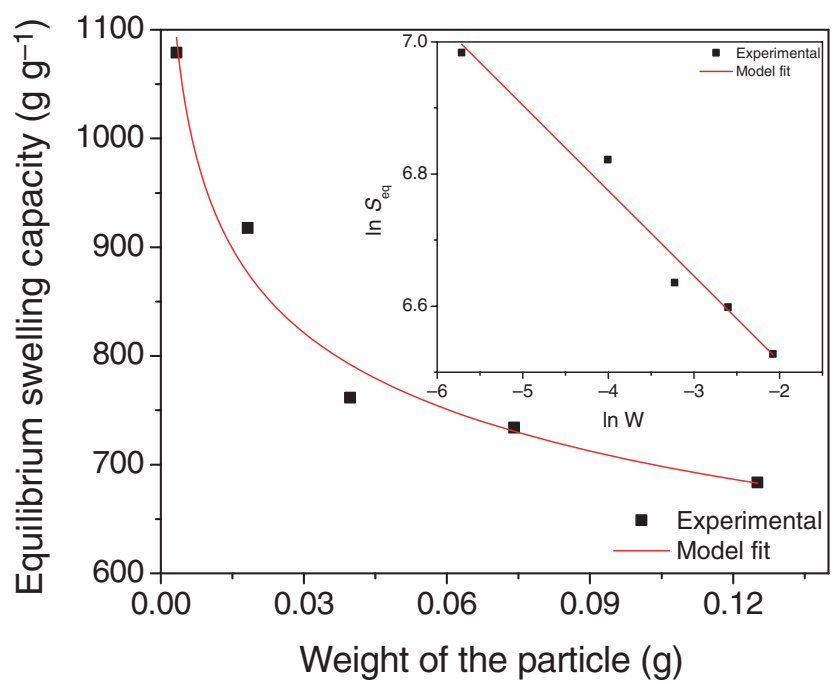

Figure 7. Variation in equilibrium swelling capacity with the size of the particle. The inset shows the linear form of the model.
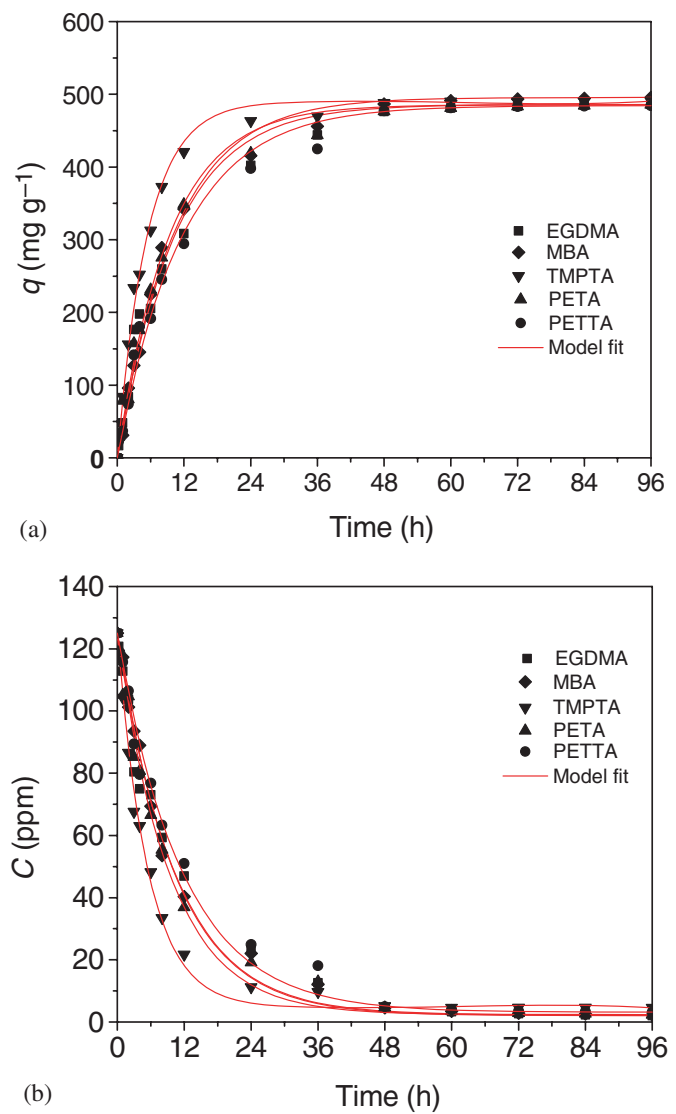

Figure 8. Variation in the (a) dye adsorption capacity and (b) concentration of OG solution at initial concentration $125 \mathrm{ppm}$ with time for different SAPs.

Figure 1 shows the change in swelling capacities for the polymers in DI water with time. The order of the swelling capacities is as follows: TMPTA > PETA > PETTA > MBA > EGDMA. The equilibrium swelling capacity first increases with increase in functionality from 4 to 6 and then
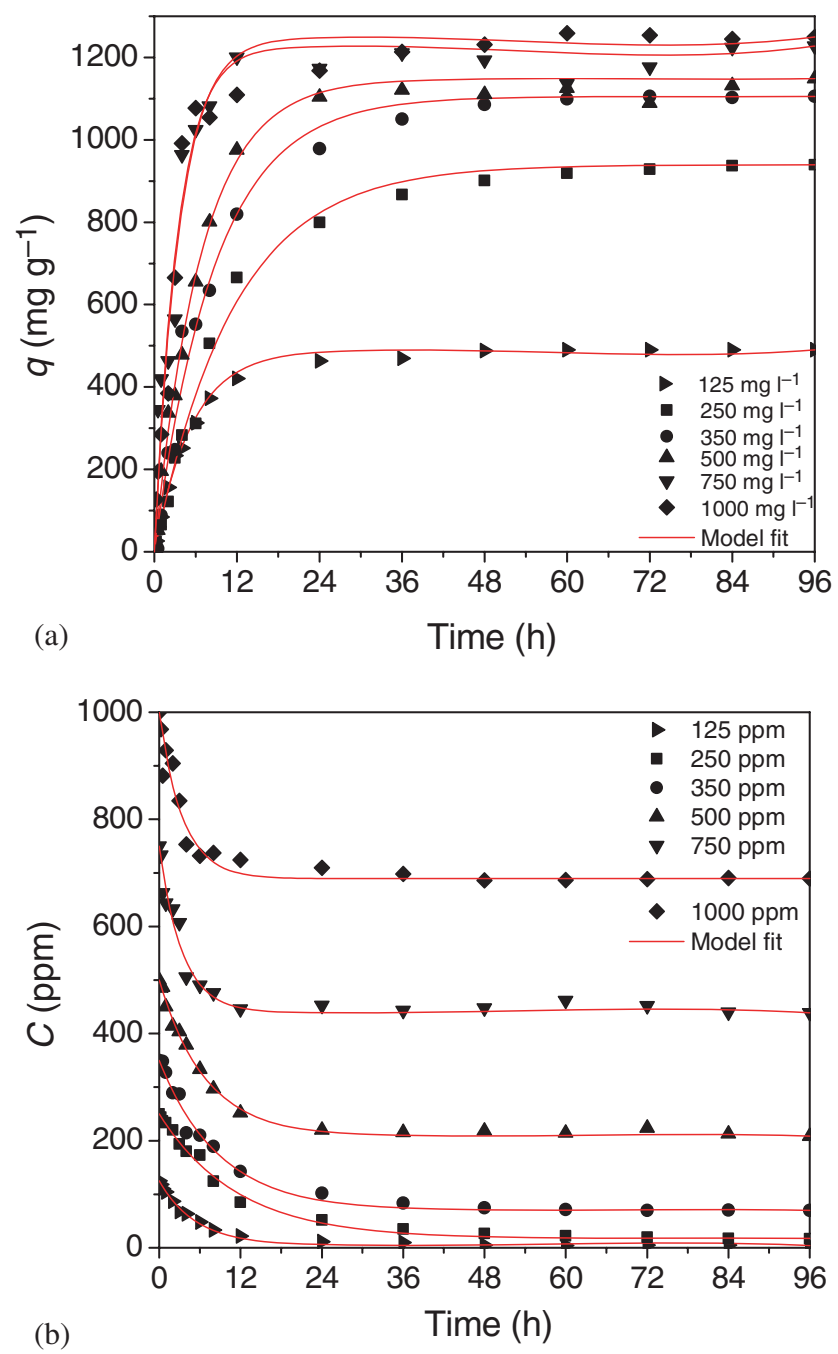

Figure 9. Variation in the (a) dye adsorption capacity and (b) concentration of OG solution for different initial dye (OG) concentrations with time for TMPTA.

decreases from 6 to 8 . Within the crosslinkers of the same functionality, there were differences in the swelling capacity. Among the tetrafunctional crosslinkers, MBA showed higher swelling capacity than EGDMA. Among the hexafunctional crosslinkers, TMPTA showed higher swelling capacity than PETA. The swelling capacity of the polymer synthesized with the octafunctional crosslinker was greater than the polymers synthesized with both the tetrafunctional crosslinkers. It would be expected that the swelling capacity would remain the same for crosslinkers with the same functionality and decrease with increase in functionality. However, this is not always seen. There are several studies in the literature that show that there is no direct relation between functionality and swelling capacity. The hydrogels of acrylamide have been prepared by chemical initiation and gamma irradiation in the presence of three tetrafunctional crosslinkers MBA, EGDMA and butanedioldimethacrylate (BDDM) as crosslinkers [20]. In the case of radiation synthesized hydrogels, polymers crosslinked with MBA and BDDM showed similar swelling capacity while those crosslinked 
with EGDMA showed a higher swelling capacity. In the case of chemical initiation method, the polymers synthesized using MBA showed lower swelling capacity as compared to EGDMA for the same amount of crosslinker concentration. The effect on the properties of acrylic-based polymers due to crosslinker has been studied [7]. The polymers were prepared by inverse suspension polymerization and they showed similar swelling capacities for MBA and EGDMA crosslinkers. However, the swelling rate of polymers crosslinked with
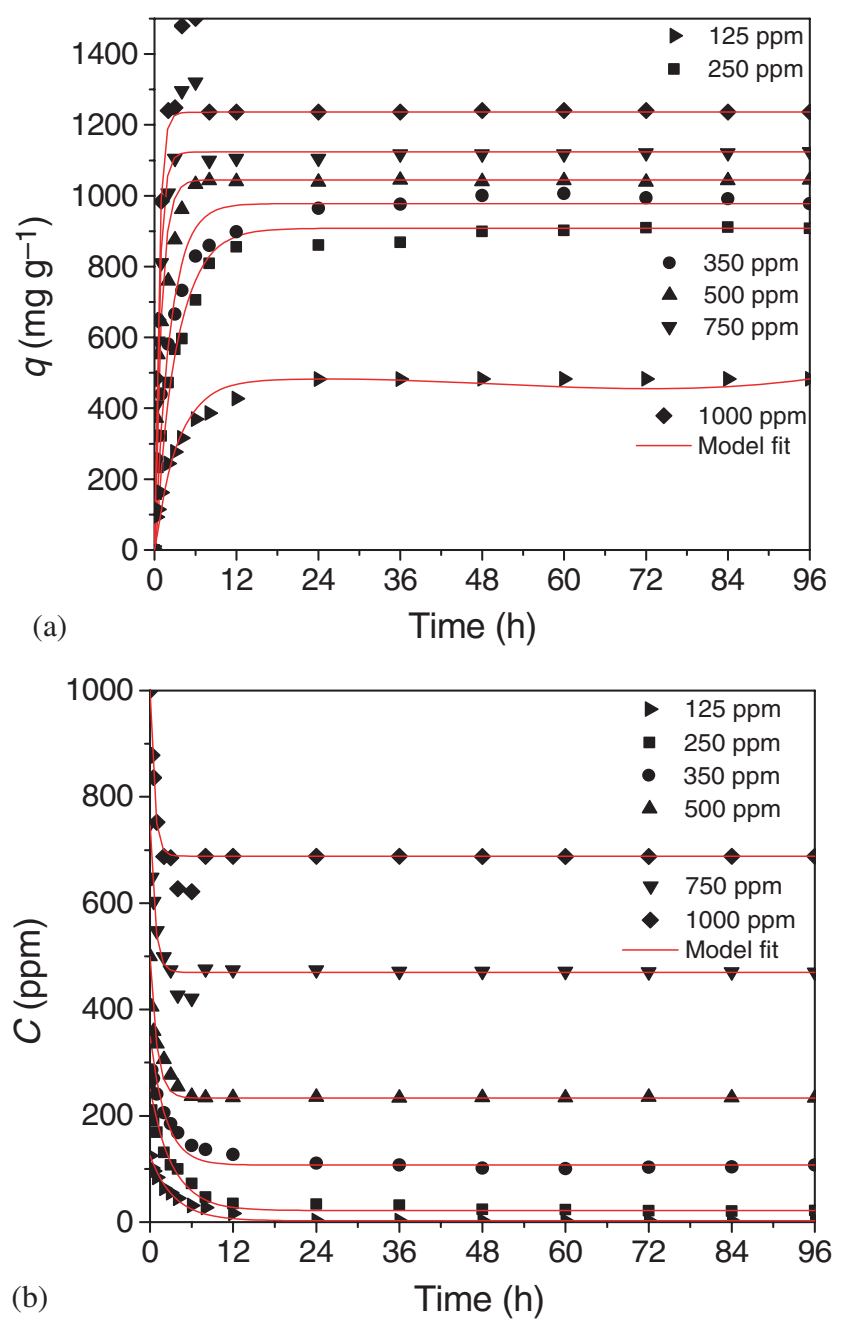

Figure 10. Variation in the (a) dye adsorption capacity and (b) concentration of OG solution for different initial dye (OG) concentrations with time for EGDMA.

Table 6. Parameters for dye adsorption.

\begin{tabular}{lccr}
\hline & \multicolumn{3}{c}{$125 \mathrm{ppm}$} \\
\cline { 2 - 4 } Polymer & $C_{\mathrm{e}}(\mathrm{ppm})$ & $q_{\mathrm{e}}\left(\mathrm{mg} \mathrm{g}^{-1}\right)$ & $k_{\mathrm{a}}\left(\mathrm{h}^{-1}\right)$ \\
\hline MBA & 2 & 495 & $0.095 \pm 0.003$ \\
EGDMA & 2 & 485 & $0.10 \pm 0.005$ \\
TMPTA & 5 & 490 & $0.18 \pm 0.006$ \\
PETA & 2 & 485 & $0.10 \pm 0.005$ \\
PETTA & 3 & 484 & $0.11 \pm 0.004$ \\
\hline
\end{tabular}

EGDMA was higher than MBA. The copolymers of $N$-vinyl2-pyrolidone and butyl acrylate using tetrafunctional MBA and hexafunctional 1,1,1 trimethylpropane trimethacrylate (TPT) by $\gamma$-irradiation have been synthesized [10]. The ones prepared with TPT showed slightly higher swelling capacity as compared to the ones prepared using MBA. However, the polymers crosslinked with TPT had higher sol fraction. Hydrogels of acrylamide and crotonic acid have been prepared using tetrafunctional 1,4-butanediol dimethacrylate (BDMA) and hexafunctional TMPTA [21]. In the absence of crotonic acid, equilibrium swelling capacity was higher for BDMA while in its presence, swelling was higher for TMPTA. The copolymers of $N$-isopropylacrylamide and acrylic acid using MBA and an octafunctional crosslinker glyoxal bis(diallyl acetal) (GLY) have been prepared by solution polymerization [8]. The swelling capacity was greater for polymers crosslinked with GLY. Thus, increasing functionality does not necessarily mean a decrease in the swelling capacity of the polymer. Apart from the functionality of the crosslinkers, there are factors such as structure of crosslinker, reactivity of the crosslinker and the monomer and method of polymerization that decide the swelling capacity of the polymers. The formation of an irregular structured polymer network is widely accepted as one explanation for discrepancies found between the predicted and observed behaviour of polymers [22].

\subsection{Swelling/de-swelling}

The polymers were subjected to cyclic swelling/de-swelling in DI water and $0.1 \mathrm{M} \mathrm{NaCl}$ solution. They were swelled in DI water and upon reaching equilibrium, they were transferred to $0.1 \mathrm{M} \mathrm{NaCl}$ solution.

When the swollen polymer is kept in a saline solution, it loses water if the concentration of ions is higher in the surrounding medium than inside. It loses water until equilibrium is reached and this swelling capacity at the equilibrium is known as the limiting swelling capacity [19].

The kinetics of the swelling and de-swelling were fitted using a first-order model. The following equation was used $[18,23]$ :

$$
S=S_{\mathrm{eq}}-\left(S_{\mathrm{eq}}-S_{0}\right) \exp \left(-k_{\mathrm{s}} t\right),
$$

where $S_{\text {eq }}$ is the equilibrium swelling capacity of the polymer. In case of de-swelling, equilibrium swelling capacity is equal to the limiting swelling capacity, $S_{\mathrm{L}}$. $S_{0}$ is the swelling capacity at the beginning of a swelling or a de-swelling cycle, where $t$ is time. $S_{0}$ is equal to zero for the first swelling cycle. The rate constant for swelling is $k_{\mathrm{s}}$, while in the case of de-swelling it is written as $k_{\mathrm{d}}$.

Figure 2 shows the swelling and de-swelling cycles 1, 2 and 3, which follow first-order kinetics. Tables 1 and 2 show the equilibrium swelling capacities and rate constants for the swelling and de-swelling cycles. The decreasing order of equilibrium swelling capacity in swelling cycle 1 is as follows: TMPTA $>$ PETA $>$ PETTA $>$ MBA $>$ 
Table 7. Parameters for the adsorption of OG by TMPTA for different initial dye (OG) concentrations.

\begin{tabular}{lcccccc}
\hline$C_{\mathrm{o}}(\mathrm{ppm})$ & 125 & 250 & 350 & 500 & 750 & 1000 \\
\hline$C_{\mathrm{e}}(\mathrm{ppm})$ & 5 & 17 & 70 & 209 & 439 & 690 \\
$q_{\mathrm{e}}\left(\mathrm{mg} \mathrm{g}^{-1}\right)$ & 490 & 940 & 1105 & 1148 & 1227 & 1249 \\
$k_{\mathrm{a}}\left(\mathrm{h}^{-1}\right)$ & $0.18 \pm 0.005$ & $0.08 \pm 0.003$ & $0.11 \pm 0.005$ & $0.15 \pm 0.004$ & $0.29 \pm 0.02$ & $0.27 \pm 0.02$ \\
\hline
\end{tabular}

Table 8. Parameters for the adsorption of OG by EGDMA for different initial dye (OG) concentrations.

\begin{tabular}{lcccccc}
\hline$C_{\mathrm{o}}(\mathrm{ppm})$ & 125 & 250 & 350 & 500 & 750 & 1000 \\
\hline$C_{\mathrm{e}}(\mathrm{ppm})$ & 6 & 33 & 125 & 270 & 510 & 753 \\
$q_{\mathrm{e}}\left(\mathrm{mg} \mathrm{g}^{-1}\right)$ & 460 & 860 & 895 & 922 & 946 & 953 \\
$k_{\mathrm{a}}\left(\mathrm{h}^{-1}\right)$ & $0.104 \pm 0.005$ & $0.115 \pm 0.005$ & $0.136 \pm 0.003$ & $0.170 \pm 0.004$ & $0.327 \pm 0.016$ & $0.439 \pm 0.033$ \\
\hline
\end{tabular}

EGDMA. During the de-swelling cycle, the polymer samples showed a rapid decrease in weight due to the loss of water into the surrounding medium having a higher concentration. The polymer samples stopped de-swelling once the activity of ions inside the polymers and in the surrounding medium were equal to each other and thus reached the limiting de-swelling capacity. There were only marginal differences between the limiting swelling capacities of the various polymers.

The swelling cycle 2 showed a similar trend in the swelling capacities of the polymer samples. The decreasing order of the swelling capacity is as follows: TMPTA $>$ PETTA $>$ PETA $>$ MBA > EGDMA. However, the difference between the swelling capacities of PETA and PETTA is not much pronounced. The polymers TMPTA and PETA showed the greatest extent of decrease in the swelling capacity as compared to that in the first cycle. There was a substantial decrease in the limiting swelling capacities of PETA and TMPTA in the de-swelling cycle 2 .

In swelling cycle 3, PETTA showed the maximum swelling capacity. The polymers TMPTA, MBA and PETA showed nearly the same swelling capacities. EGDMA showed the minimum swelling capacity. The swelling capacities of EGDMA, MBA and PETTA are nearly equal to the values they showed in the previous swelling cycle. However, PETA and TMPTA showed an appreciable decrease in swelling capacities than the values in the previous cycle. In de-swelling cycle 3, PETTA, EGDMA and MBA showed similar limiting swelling capacity. PETA and TMPTA showed the least limiting swelling capacity.

The decrease in the swelling capacity of the polymers in the subsequent cycles can be understood by two factors: diffusion of $\mathrm{NaCl}$ solution into the polymers during de-swelling [18] and dissolution of the polymer. Figure 3 shows the conductivity of the medium during the swelling cycles for EGDMA, MBA, TMPTA and PETTA. The value of the conductivity during the swelling cycles 2 and 3 is reasonably higher than that for cycle 1 for all the polymers. This implies that $\mathrm{NaCl}$ solution diffuses into the polymer matrix during de-swelling. When the polymers are swelled in the DI water in the next cycle, $\mathrm{NaCl}$ diffuses out and thus, the swelling

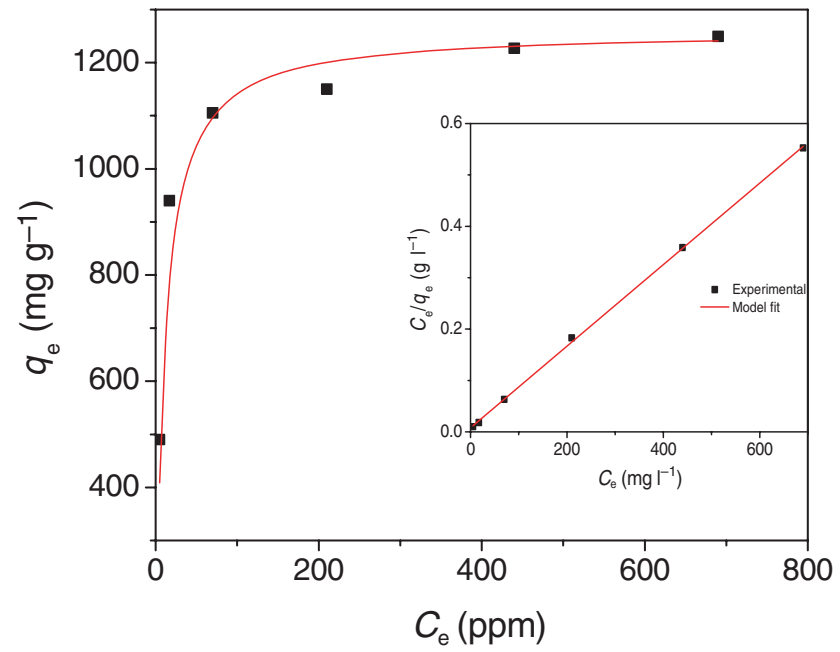

Figure 11. Langmuir adsorption isotherms for OG adsorbed on TMPTA. The inset shows the linear form of the isotherm.

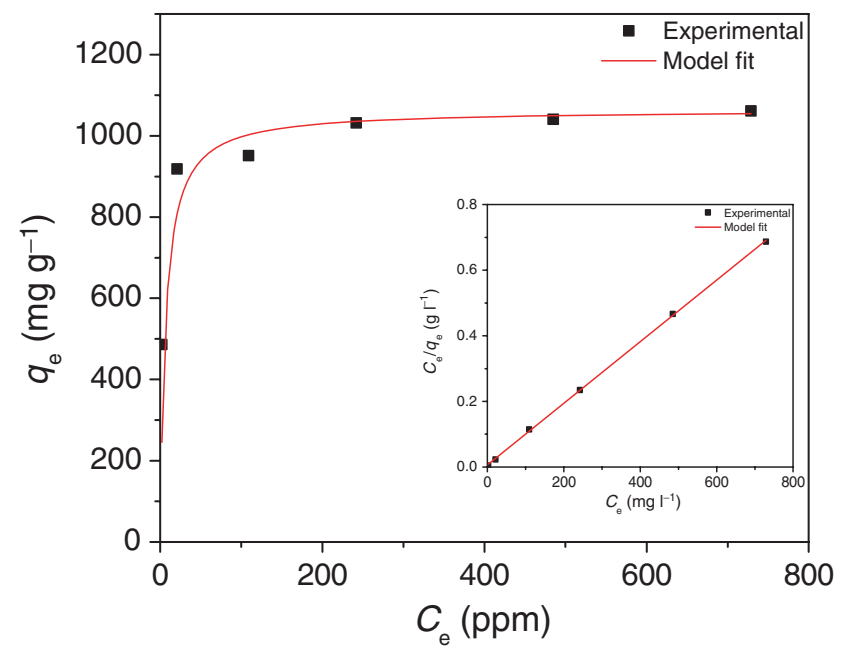

Figure 12. Langmuir adsorption isotherms for OG adsorbed on EGDMA. The inset shows the linear form of the isotherm.

medium effectively turns into an $\mathrm{NaCl}$ solution [18]. This diffusion of $\mathrm{NaCl}$ was verified by monitoring the conductivity 
of the swelling/de-swelling medium. The amount of the $\mathrm{NaCl}$ released in the swelling medium during swelling cycles 2 and 3 was found to be almost the same, as confirmed by the conductivity measurements. This reduces the osmotic pressure difference and thus the swelling capacity of the polymers reduces in the second and the third cycles. The values of conductivity in the third cycle are almost same as that in the second cycle for EGDMA and decrease only slightly for MBA and PETTA. The values of swelling capacities in the second and the third cycles are nearly the same for these polymers. The values of conductivity are visibly lower in the third cycle than the second cycle for TMPTA and PETTA. Thus the conductivity measurements correlate with the swelling capacities. Even then the swelling capacities are appreciably lower in the third cycle for these polymers. This could be due to the dissolution of polymer chains during the various cycles. To illustrate this, the soluble fraction of the polymer samples was determined by the procedure mentioned in section 2.7. The sol fraction, as calculated by equation 1 , of the polymers EGDMA, MBA, TMPTA, PETA and PETTA was
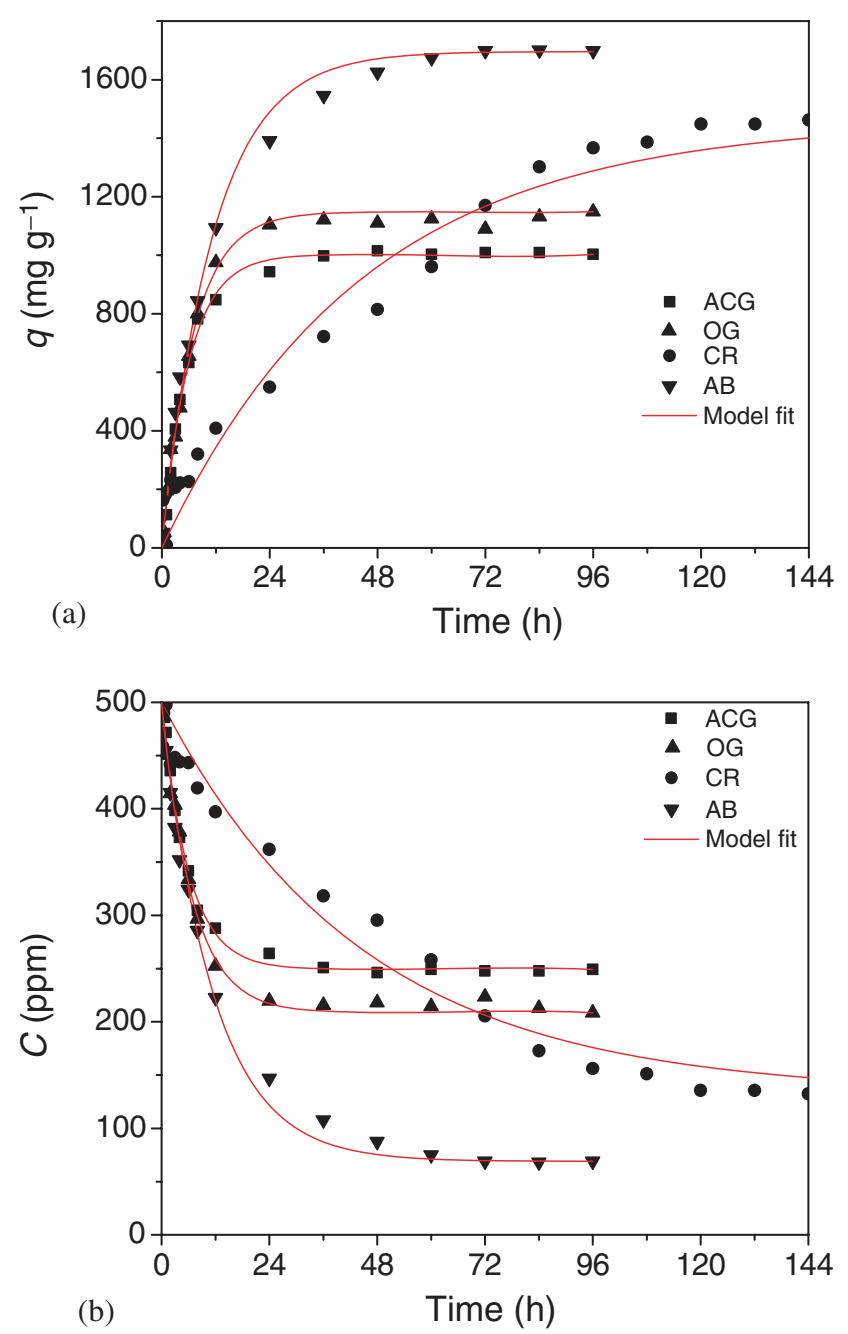

Figure 13. Variation in the (a) dye adsorption capacity and (b) concentration of the dye solutions with time for TMPTA.
$0.202,0.217,0.395,0.309$ and 0.236 , respectively. It is clear that the TMPTA and PETA have the highest soluble content, while EGDMA, MBA and PETTA have low soluble content. It is in agreement with the extent of decrease in swelling capacities of the polymers.

There were only marginal differences in the limiting swelling capacities despite the large differences in the swelling capacities in DI water. To verify this, the polymers were swelled in $0.1 \mathrm{M} \mathrm{NaCl}$ solution. This is discussed in the next section.

\subsection{Swelling in saline solution}

Figures 4 and 5 show the variation of swelling capacity in 0.1 and $0.05 \mathrm{M} \mathrm{NaCl}$ solutions, respectively, with time for the polymers. Table 3 shows the comparison between the swelling capacity and the limiting swelling capacity of the polymers for $0.1 \mathrm{M} \mathrm{NaCl}$. Table 4 shows the swelling capacity in $0.05 \mathrm{M} \mathrm{NaCl}$ solution. They show similar trends. There are slight differences between the swelling capacities of the
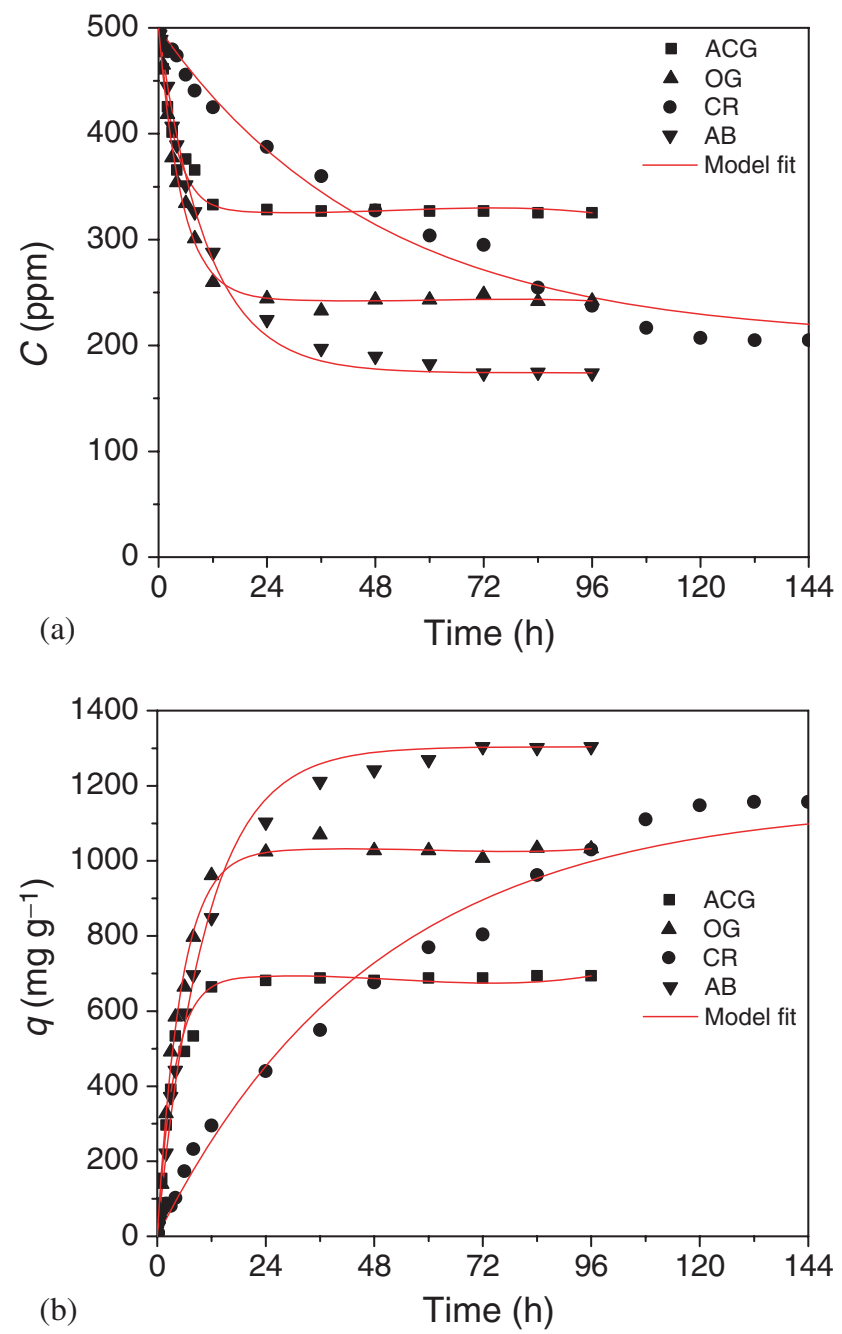

Figure 14. Variation in the (a) dye adsorption capacity and (b) concentration of the dye solutions for EGDMA. 
Table 9. Parameters of dye adsorption for different dyes by TMPTA.

\begin{tabular}{lccccc}
\hline Dye & $C_{\mathrm{e}}(\mathrm{ppm})$ & $q_{\mathrm{e}}\left(\mathrm{mg} \mathrm{g}^{-1}\right)$ & $k_{\mathrm{a}}\left(\mathrm{h}^{-1}\right)$ & $\mathrm{MW}\left(\mathrm{g} \mathrm{mol}^{-1}\right)$ & Molecular size $\left(\AA^{3}\right)$ \\
\hline OG & 209 & 1148 & $0.146 \pm 0.005$ & 452.38 & $13.08 \times 7.53 \times 4.98$ \\
ACG & 249 & 1003 & $0.167 \pm 0.005$ & 622.59 & $12.06 \times 12.82 \times 4.42$ \\
CR & 133 & 1458 & $0.022 \pm 0.001$ & 696.67 & $26.56 \times 7.19 \times 4.37$ \\
AB & 69 & 1697 & $0.088 \pm 0.003$ & 616.5 & $20.28 \times 8.42 \times 3.2$ \\
\hline
\end{tabular}

Table 10. Parameters of dye adsorption for different dyes by EGDMA.

\begin{tabular}{lccccc}
\hline Dye & $C_{\mathrm{e}}(\mathrm{ppm})$ & $q_{\mathrm{e}}\left(\mathrm{mg} \mathrm{g}^{-1}\right)$ & $k_{\mathrm{a}}\left(\mathrm{h}^{-1}\right)$ & $\mathrm{MW}\left(\mathrm{g} \mathrm{mol}^{-1}\right)$ & Molecular size $\left(\AA^{3}\right)$ \\
\hline OG & 242 & 1032 & $0.193 \pm 0.006$ & 452.38 & $13.08 \times 7.53 \times 4.98$ \\
ACG & 325 & 693 & $0.262 \pm 0.014$ & 622.59 & $12.06 \times 12.82 \times 4.42$ \\
CR & 205 & 1157 & $0.021 \pm 0.001$ & 696.67 & $26.56 \times 7.19 \times 4.37$ \\
AB & 174 & 1304 & $0.093 \pm 0.001$ & 616.5 & $20.28 \times 8.42 \times 3.2$ \\
\hline
\end{tabular}

polymers. There is an increase in the swelling capacity of the polymers with decrease in the concentration of the saline solution and the differences are more pronounced as compared to the earlier case. The trend in the swelling capacity of the polymers is as follows: TMPTA $>$ PETA $>$ PETTA $>$ MBA > EGDMA. It is the same as the trend seen in case of DI water.

\subsection{Effect of crosslinker concentration}

The TMPTA polymer was synthesized for different concentrations of the crosslinker, TMPTA. The TMPTA concentration was varied from $0.125 \mathrm{~mol} \%$ (of monomer) to 1 mol\% (of monomer). The swelling process followed firstorder kinetics. Figure 6 shows the variation in the swelling capacity for the polymers. The swelling capacity decreases with an increase in the crosslinker concentration. On the other hand, there is an increase in the swelling rate with an increase in the crosslinker concentration. The increase in the crosslinks makes the polymer matrix more compact and reduces the water absorption capacity. However, there is a drastic decrease in the swelling capacity from 0.125 to $0.25 \mathrm{~mol} \%$, which indicates uniform and better crosslinking at higher concentrations. Table 5 shows the equilibrium swelling capacities and rate constants for different crosslinker concentrations.

\subsection{Effect of size on equilibrium swelling capacity}

To investigate the effect of size on the swelling capacity, TMPTA polymer particles of different weights were taken and they were allowed to swell in DI water for $24 \mathrm{~h}$. Figure 7 shows that the swelling capacity increases with decrease in the size of the particles. It is suggested that the increase in surface area of the particles with decreasing size leads to higher swelling capacities [24]. The relation between the equilibrium swelling capacity and the weight of the particle
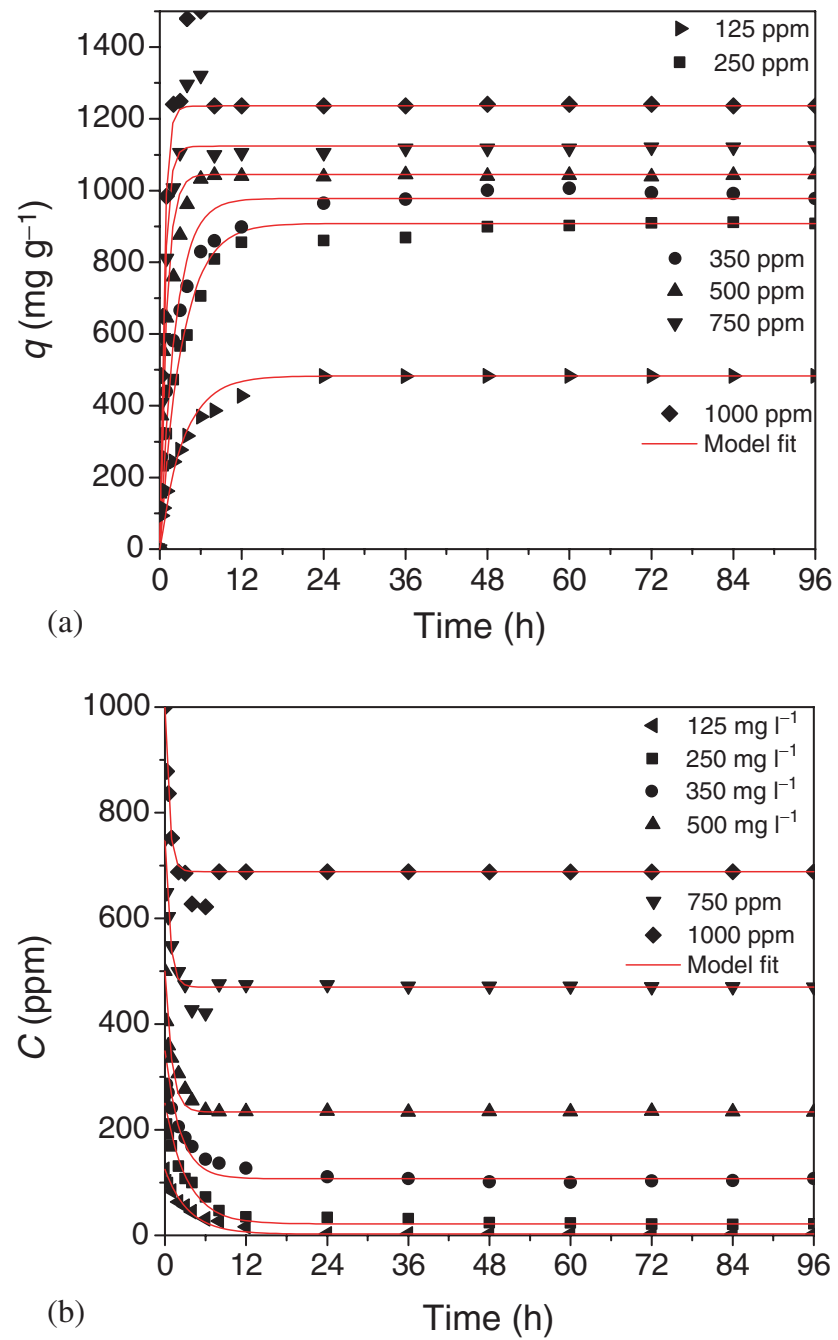

Figure 15. Variation in the (a) dye adsorption capacity and (b) concentration of OG solution for different initial dye (OG) concentrations with time for swollen EGDMA.

can be written as, $S_{\mathrm{eq}}=\mathrm{CW}^{n}$. Therefore, a log-log plot of $S_{\text {eq }}$ with weight is expected to be linear. 


\subsection{Dye adsorption}

The polymer poly(METAC) consists of cationic repeat units and can be used for the adsorption of oppositely charged dyes by electrostatic attraction. During adsorption, the polymer appears coloured due to the dye particles adsorbed on the surface while the dye solution becomes clearer. The dye adsorption capacity for OG was determined for the polymers
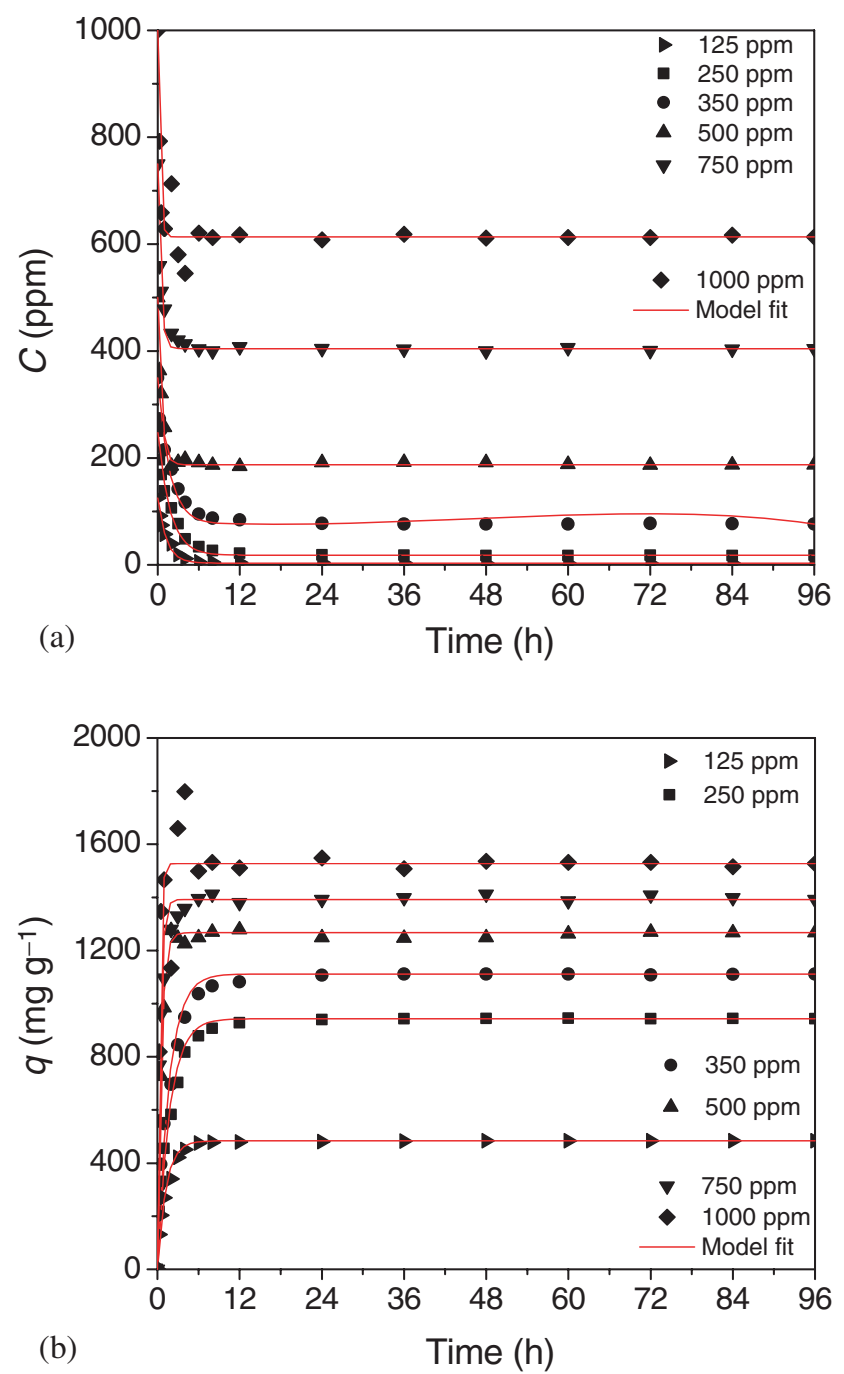

Figure 16. Variation in the (a) dye adsorption capacity and (b) concentration of OG solution for different initial dye (OG) concentrations with time for swollen TMPTA.
EGDMA, MBA, TMPTA, PETA and PETTA at an initial concentration of $125 \mathrm{ppm}$. The adsorption capacity of TMPTA was checked at different initial concentrations. Apart from $\mathrm{OG}$, three other dyes $\mathrm{ACG}, \mathrm{CR}$ and $\mathrm{AB}$ were used for adsorption on TMPTA at an initial concentration of $500 \mathrm{ppm}$.

3.6a Kinetics of adsorption: The dye adsorption followed first-order kinetics satisfactorily. The following equation, after integration, was used for fitting [18]:

$$
\frac{\mathrm{d} q}{\mathrm{~d} t}=k_{\mathrm{a}}\left(q_{\mathrm{e}}-q\right)
$$

where $q$ is the weight of dye adsorbed per unit weight of the dry polymer and $q_{\mathrm{e}}$ the weight of the dye adsorbed per unit weight of the dry polymer at equilibrium. The initial condition for the above equation is, at $t=0, q=0$. On integrating the equation, the following expression is obtained:

$$
q=q_{\mathrm{e}}\left(1-\exp \left(-k_{\mathrm{a}} t\right)\right) .
$$

3.6b Effect of crosslinker on dye adsorption: The same amount of different SAPs was brought in contact with dye solutions of the same initial concentration to determine the effect of crosslinker on the dye adsorption capacity of the polymer. The experiments were carried out at $125 \mathrm{ppm}$. Figure 8 shows the change in the amount of dye adsorbed on the SAP and change in the concentration of dye solution with time. Table 5 shows the kinetic parameters associated with the dye adsorption. The polymers show nearly same dye adsorption capacities due to the low concentration of the dye. The concentration of the sites for adsorption may be larger than the concentration of dye particles in the solution for all the polymers and thus show similar adsorption capacities. To have a comparative study, polymers TMPTA and EGDMA were used to adsorb dyes at higher concentrations.

\subsection{Effect of initial dye concentration}

The polymers EGDMA and TMPTA were used to adsorb OG at different initial concentrations: 125, 250, 350, 500, 750 and 1000 ppm. Figures 9 and 10 show the variation in the concentration of the dye solution and dye adsorbed

\begin{tabular}{|c|c|c|c|c|c|c|}
\hline \multirow[b]{2}{*}{ Conc. (ppm) } & \multicolumn{3}{|c|}{ Unswollen } & \multicolumn{3}{|c|}{ Swollen } \\
\hline & $C_{\mathrm{e}}(\mathrm{ppm})$ & $q_{\mathrm{e}}\left(\mathrm{mg} \mathrm{g}^{-1}\right)$ & $k_{\mathrm{a}}\left(\mathrm{h}^{-1}\right)$ & $C_{\mathrm{e}}(\mathrm{ppm})$ & $q_{\mathrm{e}}\left(\mathrm{mg} \mathrm{g}^{-1}\right)$ & $k_{\mathrm{a}}\left(\mathrm{h}^{-1}\right)$ \\
\hline 125 & 2 & 486 & $0.091 \pm 0.002$ & 3 & 483 & $0.29 \pm 0.02$ \\
\hline 250 & 21 & 918 & $0.093 \pm 0.003$ & 22 & 908 & $0.305 \pm 0.028$ \\
\hline 350 & 109 & 951 & $0.132 \pm 0.004$ & 107 & 978 & $0.44 \pm 0.04$ \\
\hline 500 & 242 & 1032 & $0.190 \pm 0.005$ & 234 & 1045 & $1.01 \pm 0.11$ \\
\hline 750 & 485 & 1040 & $0.277 \pm 0.008$ & 470 & 1123 & $1.44 \pm 0.16$ \\
\hline 1000 & 729 & 1061 & $0.372 \pm 0.021$ & 689 & 1236 & $1.7 \pm 0.2$ \\
\hline
\end{tabular}

Table 11. Parameters for dye adsorption for different dyes by swollen EGDMA. 
Table 12. Parameters of dye adsorption for different dyes by swollen TMPTA.

\begin{tabular}{lccccccc}
\hline & \multicolumn{3}{c}{ Unswollen } & & \multicolumn{3}{c}{ Swollen } \\
\cline { 2 - 3 } Conc. $(\mathrm{ppm})$ & $C_{\mathrm{e}}(\mathrm{ppm})$ & $q_{\mathrm{e}}\left(\mathrm{mg} \mathrm{g}^{-1}\right)$ & $k_{\mathrm{a}}\left(\mathrm{h}^{-1}\right)$ & & $C_{\mathrm{e}}(\mathrm{ppm})$ & $q_{\mathrm{e}}\left(\mathrm{mg} \mathrm{g}^{-1}\right)$ & $k_{\mathrm{a}}\left(\mathrm{h}^{-1}\right)$ \\
\hline 125 & 5 & 490 & $0.18 \pm 0.005$ & 3 & 484 & $0.798 \pm 0.052$ \\
250 & 17 & 940 & $0.08 \pm 0.003$ & & 18 & 943 & $0.558 \pm 0.038$ \\
350 & 70 & 1105 & $0.110 \pm 0.005$ & 77 & 1112 & $0.581 \pm 0.047$ \\
500 & 209 & 1148 & $0.150 \pm 0.004$ & & 209 & 1163 & $1.798 \pm 0.09$ \\
750 & 439 & 1227 & $0.29 \pm 0.02$ & & 405 & 1392 & $2.404 \pm 0.206$ \\
1000 & 690 & 1249 & $0.27 \pm 0.02$ & & 613 & 1528 & $3.459 \pm 0.591$ \\
\hline
\end{tabular}

by the adsorbents TMPTA and EGDMA, respectively, with time. Table 6 shows the parameters of dye adsorption. The total amount of dye adsorbed by the polymer increases with an increase in the initial dye concentration upto a certain extent and saturates subsequently due to the saturation of all the available sites after a certain concentration. The various parameters associated with dye adsorption are shown in tables 7 and 8 for TMPTA and EGDMA, respectively. The amount of dye adsorbed on the two polymers is comparable at lower concentrations. However, as the concentration increases the difference becomes appreciable but it is not too high.

\subsection{Adsorption isotherm}

Langmuir isotherm was used to describe the adsorption. It is given by the following equation:

$$
q_{\mathrm{e}}=\frac{q_{\mathrm{m}} K C_{\mathrm{e}}}{1+K C_{\mathrm{e}}} .
$$

The linear form of the Langmuir adsorption isotherm is given as follows:

$$
\frac{C_{\mathrm{e}}}{q_{\mathrm{e}}}=\frac{C_{\mathrm{e}}}{q_{\mathrm{m}}}+\frac{1}{q_{\mathrm{m}} K},
$$

where $C_{\mathrm{e}}$ is the equilibrium adsorbate concentration (ppm), $q_{\mathrm{e}}$ the quantity of adsorbate adsorbed at equilibrium per unit mass of the adsorbent $\left(\mathrm{mg} \mathrm{g}^{-1}\right), q_{\mathrm{m}}$ the maximum amount of the adsorbate adsorbed per unit mass of the adsorbent $\left(\mathrm{mg} \mathrm{g}^{-1}\right)$ and $K$ the Langmuir adsorption constant $\left(\mathrm{mg}^{-1}\right)$.

Figures 11 and 12 show the Langmuir adsorption isotherm for the dye OG adsorbed using the TMPTA and EGDMA polymer, respectively. The values of the constants $q_{\mathrm{m}}$ and $K$ are $1260 \mathrm{mg} \mathrm{g}^{-1}$ and $0.0961 \mathrm{mg}^{-1}$ for TMPTA and $1064 \mathrm{mg} \mathrm{g}^{-1}$ and $0.15 \mathrm{l} \mathrm{mg}^{-1}$ for EGDMA.

\subsection{Adsorption of various dyes}

The TMPTA and EGDMA polymers were used to adsorb three other dyes $\mathrm{ACG}, \mathrm{CR}$ and $\mathrm{AB}$ at initial concentration of $500 \mathrm{ppm}$. Figures 13 and 14 show a comparison between the variation in the concentration of dye solution and dye adsorbed per unit weight of the adsorbent for different dyes for TMPTA and EGDMA, respectively. The decreasing order
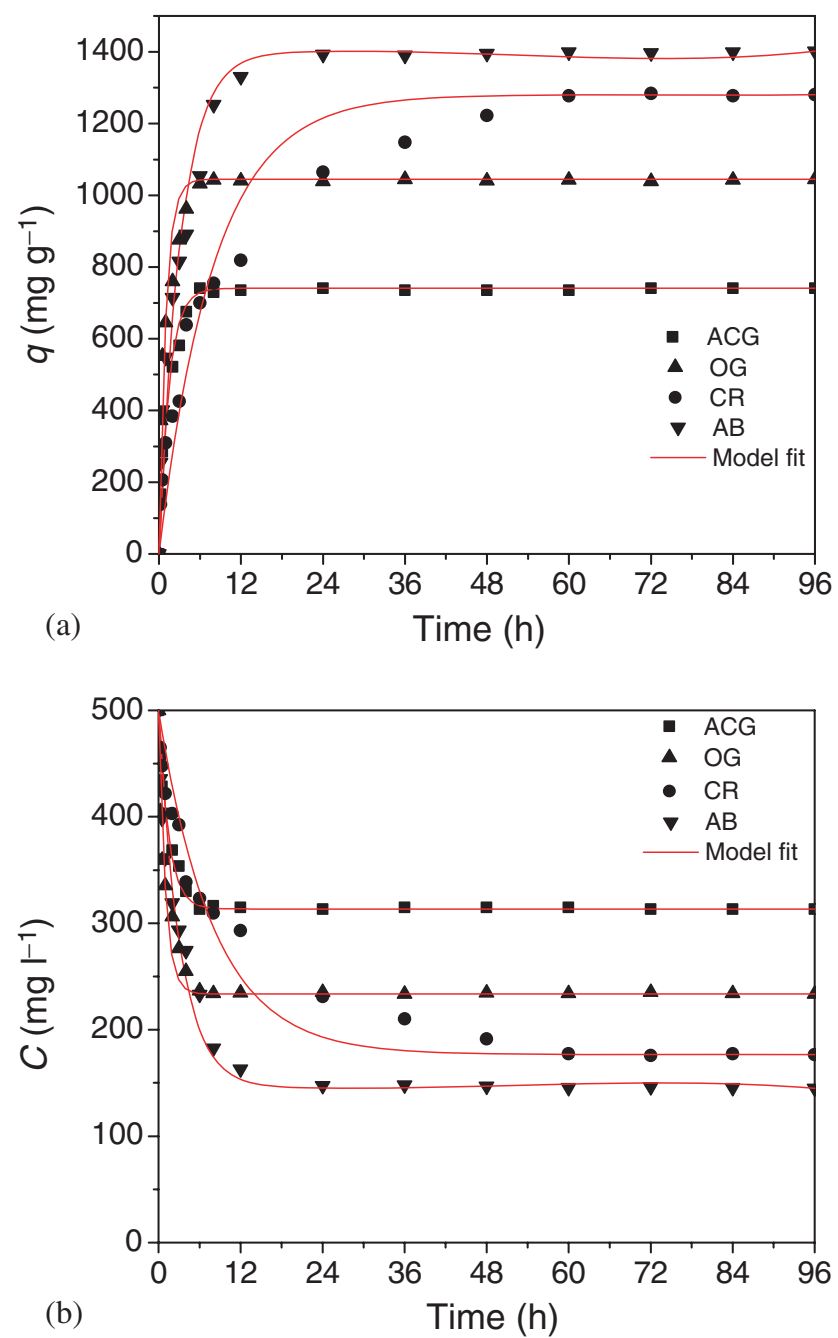

Figure 17. Variation in the (a) dye adsorption capacity and (b) concentration of the dye solutions with time for swollen EGDMA.

of the dye adsorption capacity at equilibrium is as follows: TMPTA, AB > CR > OG > ACG and EGDMA, AB > CR $>$ OG $>$ ACG. Tables 9 and 10 show the final concentration of the dye solution, dye adsorption capacity of the dye solution and the rate constant for TMPTA and EGDMA, respectively. The dye adsorption capacity of TMPTA is higher than EGDMA for all the dyes. The dyes consist of different functional groups and have different molecular weights and 
sizes. All these factors can be attributed to the differences in the dye adsorption capacity and the rate of adsorption for the dyes.

\subsection{Adsorption of dye on swollen polymer}

The polymers EGDMA and TMPTA were swollen to their equilibrium swelling capacity and the swollen polymers were used to adsorb the dyes.
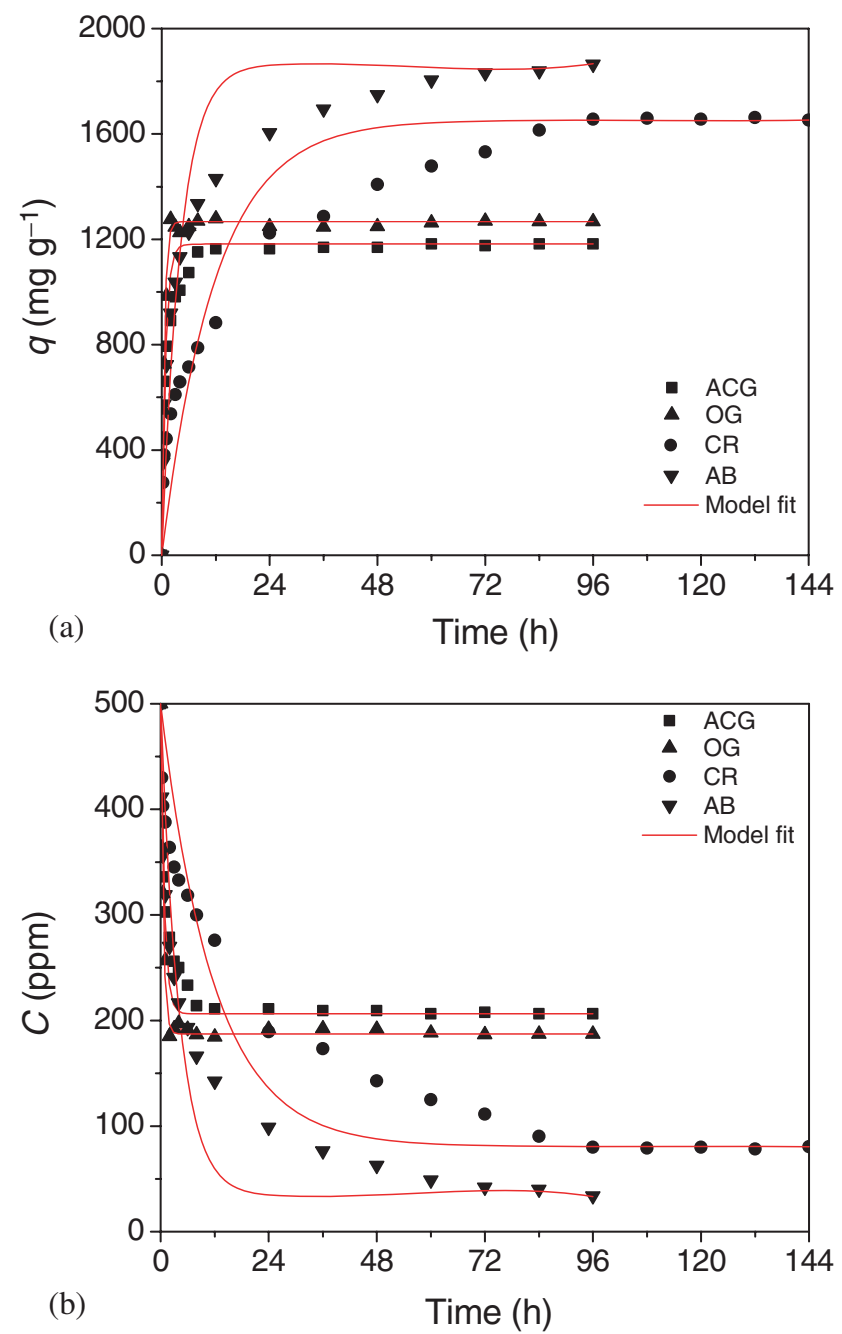

Figure 18. Variation in the (a) dye adsorption capacity and (b) concentration of the dye solutions with time for swollen TMPTA.

\subsection{Effect of initial dye concentration}

The swollen polymers of TMPTA and EGDMA were used for adsorbing dye orange $\mathrm{G}$. The solutions of the dye were of the following concentration: 125, 250, 350, 500, 750 and $1000 \mathrm{ppm}$. Figures 15 and 16 show the variation in the dye adsorption capacity and the concentration of the dye solution with time for EGDMA and TMPTA, respectively. As is evident from the graphs, the rate of adsorption is quite high. Tables 11 and 12 show the comparison of various parameters of adsorption with unswollen and swollen polymers for EGDMA and TMPTA, respectively. In both the cases, the rate constants are quite high for swollen polymers as compared to the unswollen polymers. The rate constants for swollen TPMTA are even higher as compared to swollen EGDMA. The dye adsorption capacity seems to increase with increase in concentration for both the swollen polymers, while it reaches saturation if they are unswollen.

In case of dye adsorption with unswollen polymer, the polymer swells during the process. Thus, the sites become available for adsorption gradually. The swelling capacity of the polymers differs with dye solutions due to differences in the $\mathrm{pH}$ of different solutions. It is appreciably lesser than DI water. The polymers are swollen in DI water. When the polymer is in the swollen state, the sites are available for adsorption from the beginning resulting in high rates of adsorption. However, the polymers lose water due to the $\mathrm{pH}$ of the dye solution. The quantity of water given to the solution by the polymers is appreciable. Therefore, even though the dye adsorption capacity should reach saturation, it seems to increase with increase in initial dye concentration because the formula for calculating it does not incorporate the changes in the volume of water. The extent of increase is higher in case of TMPTA than EGDMA, because it releases more water than EGDMA due to its higher water absorption capacity.

\subsection{Adsorption of various dyes}

The swollen polymers of EGDMA and TMPTA were used to adsorb three other dyes ACG, AB and CR. Figures 17 and 18 show the variation of dye adsorption capacity and the concentration of solution with time for EGDMA and TMPTA. Tables 13 and 14 show the comparison between various parameters of dye adsorption for unswollen and swollen polymers of EGDMA and TMPTA, respectively. The decreasing order of dye adsorption capacity is as follows: EGDMA, $\mathrm{AB}>\mathrm{CR}>\mathrm{OG}>\mathrm{ACG}$ and TMPTA, $\mathrm{AB}>$

Table 13. Parameters of dye adsorption for different dyes by swollen EGDMA.

\begin{tabular}{|c|c|c|c|c|c|c|}
\hline \multirow[b]{2}{*}{ Dye } & \multicolumn{3}{|c|}{ Unswollen } & \multicolumn{3}{|c|}{ Swollen } \\
\hline & $C_{\mathrm{e}}(\mathrm{ppm})$ & $q_{\mathrm{e}}\left(\mathrm{mg} \mathrm{g}^{-1}\right)$ & $k_{\mathrm{a}}\left(\mathrm{h}^{-1}\right)$ & $C_{\mathrm{e}}(\mathrm{ppm})$ & $q_{\mathrm{e}}\left(\mathrm{mg} \mathrm{g}^{-1}\right)$ & $k_{\mathrm{a}}\left(\mathrm{h}^{-1}\right)$ \\
\hline OG & 242 & 1032 & $0.193 \pm 0.006$ & 234 & 1045 & $1.01 \pm 0.11$ \\
\hline ACG & 325 & 693 & $0.262 \pm 0.014$ & 313 & 741 & $0.685 \pm 0.041$ \\
\hline $\mathrm{CR}$ & 205 & 1157 & $0.021 \pm 0.001$ & 178 & 1280 & $0.124 \pm 0.012$ \\
\hline $\mathrm{AB}$ & 174 & 1304 & $0.093 \pm 0.001$ & 152 & 1374 & $0.309 \pm 0.025$ \\
\hline
\end{tabular}


Table 14. Parameters of dye adsorption for different dyes by swollen TMPTA.

\begin{tabular}{|c|c|c|c|c|c|c|}
\hline \multirow[b]{2}{*}{ Dye } & \multicolumn{3}{|c|}{ Unswollen } & \multicolumn{3}{|c|}{ Swollen } \\
\hline & $C_{\mathrm{e}}(\mathrm{ppm})$ & $q_{\mathrm{e}}\left(\mathrm{mg} \mathrm{g}^{-1}\right)$ & $k_{\mathrm{a}}\left(\mathrm{h}^{-1}\right)$ & $C_{\mathrm{e}}(\mathrm{ppm})$ & $q_{\mathrm{e}}\left(\mathrm{mg} \mathrm{g}^{-1}\right)$ & $k_{\mathrm{a}}\left(\mathrm{h}^{-1}\right)$ \\
\hline OG & 209 & 1148 & $0.146 \pm 0.005$ & 187 & 1268 & $1.798 \pm 0.09$ \\
\hline $\mathrm{ACG}$ & 249 & 1003 & $0.167 \pm 0.005$ & 206 & 1182 & $1.193 \pm 0.161$ \\
\hline CR & 133 & 1458 & $0.022 \pm 0.001$ & 81 & 1653 & $0.084 \pm 0.012$ \\
\hline $\mathrm{AB}$ & 69 & 1697 & $0.088 \pm 0.003$ & 33 & 1867 & $0.24 \pm 0.033$ \\
\hline
\end{tabular}

$\mathrm{CR}>\mathrm{OG}>\mathrm{ACG}$. The order is same as that for unswollen polymers. The rate of dye adsorption for swollen polymers is higher than unswollen polymers due to the reasons stated in the previous sections. Similarly, as in the previous section, the dye adsorption capacity appears higher in the swollen state.

\section{Conclusions}

Cationic SAPs of METAC were synthesized by using crosslinkers of different functionalities to study the effect of crosslinkers on the swelling, adsorption properties of the polymers. They were subjected to repeated cycles of swelling and de-swelling. In the first swelling cycle, the polymers crosslinked with hexafunctional crosslinkers showed the maximum swelling. There was a decrease in the swelling capacities of all the polymers in the subsequent cycles due to diffusion of $\mathrm{NaCl}$ solution in the matrix during de-swelling cycles. The $\mathrm{NaCl}$ solution diffused out of the polymer matrix during swelling cycle and reduced the osmotic pressure difference. However, the polymers crosslinked with hexafunctional crosslinkers showed a significantly higher loss in the swelling capacity than the other polymers. The sol fraction study showed that they had the highest soluble content. The dye adsorption capacity of the polymers was found to be same at low concentration of the dye. TMPTA and EGDMA were used to adsorb four different dyes. TMPTA showed higher adsorption capacity for all the dyes. The kinetics of dye adsorption was shown to be much higher if swollen polymers were used instead of unswollen polymers.

\section{Acknowledgements}

We thank the Department of Science and Technology for the financial support and JC Bose fellowship.

\section{References}

[1] Güven O, Şen M, Karadağ E and Saraydın D 1999 Radiat. Phys. Chem. 56381
[2] Laftah W A, Hashim S and Ibrahim A N 2011 Polym.-Plast. Technol. Eng. 501475

[3] Zohuriaan-Mehr M J and Kabiri K 2008 Iran. Poly. J. 17451

[4] El-Rehim H A A, Hegazy E-S A and El-Mohdy H L A 2004 J. Appl. Polym. Sci. 931360

[5] Basta A H, El-Saied H, El-Hadi O and El-Dewiny C 2013 Polym.-Plast. Technol. Eng. 521074

[6] Marandi G B, Kermani Z P and Kurdtabar M 2013 Polym. -Plast. Technol. Eng. 52310

[7] Chen X P, Shan G R, Huang J, Huang Z M and Weng Z X 2004 J. Appl. Polym. Sci. 92619

[8] Xue W, Champ S and Huglin M B 2001 Polymer 423665

[9] Atta A M, Abdel-Bary E, Rezk K and Abdel-Azim A $2009 \mathrm{~J}$. Appl. Polym. Sci. 112114

[10] Atta A M and Abdel-Azim A A A 1998 Polym. Adv. Technol. 9340

[11] Rodríguez E and Katime I 2003 J. Appl. Polym. Sci. 90530

[12] Tang S C, Yin K and Lo I 2011 J. Contam. Hydrol. 12539

[13] Sari M 2010 Water Sci. Technol. 612097

[14] Yan L, Shuai Q, Gong X, Gu Q and Yu H 2009 CLEAN-Soil, Air, Water 37392

[15] Karadag E, Üzüm Ö B and Saraydin D 2002 Eur. Polym. J. 38 2133

[16] Li S, Yan S and Yu J 2011 Polym.-Plast. Technol. Eng. 50783

[17] Rao P, Lo I M C, Yin K and Tang S C N 2011 J. Environ. Manag. 921690

[18] Shukla N B and Madras G 2013 J. Appl. Poly. Sci. 127 2251

[19] Flory P J 1953 Principles of polymer chemistry (Ithaca, NY: Cornell University Press)

[20] Saraydın D, Karadag E, Işıkver Y, Şahiner N and Güven O 2004 J. Macromol. Sci. Part A 41419

[21] Karadağ E and Saraydın D 2002 Polym. Bull. (Berlin) 48 299

[22] Patras G, Qiao G G and Solomon D H 2001 Macromolecules 346396

[23] Yao K J and Zhou W J 1994 J. Appl. Polym. Sci. 531533

[24] Omidian H, Hashemi S, Sammes P and Meldrum I 1999 Polymer 401753 